\title{
Isolation and Characterization of Antifungal Metabolites from the Melia azedarach-Associated Fungus Diaporthe eucalyptorum
}

Yu-Qi Gao, ${ }^{\S}$ Shuang-Tian Du, ${ }^{\S}$ Jian Xiao, Da-Cheng Wang, Wen-Bo Han, Qiang Zhang,* and Jin-Ming Gao*

Shaanxi Key Laboratory of Natural Products \& Chemical Biology, College of Chemistry \& Pharmacy, Northwest A\&F University, Yangling 712100, People’s Republic of China

\section{Content}

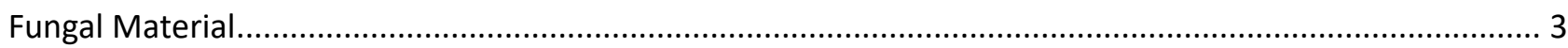

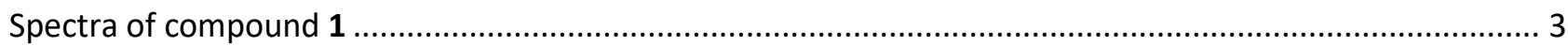

${ }^{1}$ H NMR

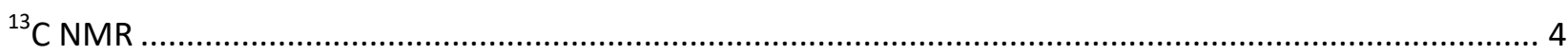

HSQC

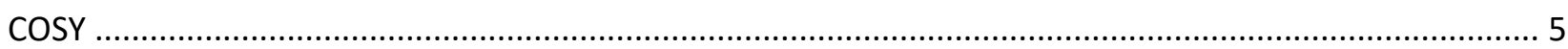

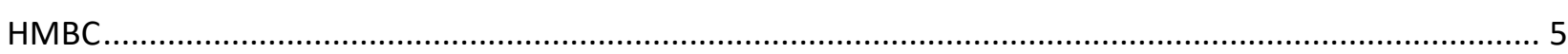

HRMS

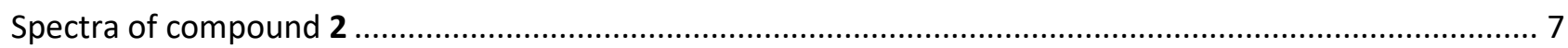

${ }^{1}$ H NMR

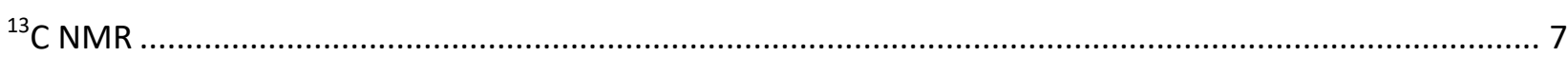

HSQC

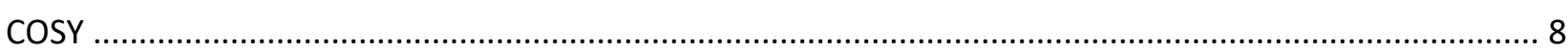

HMBC

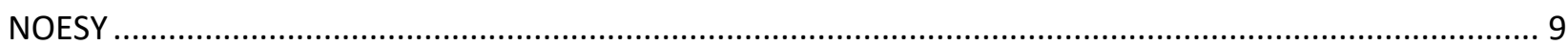

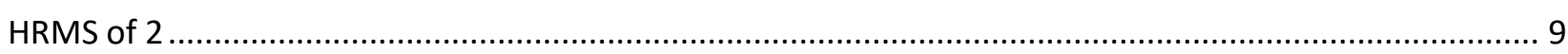

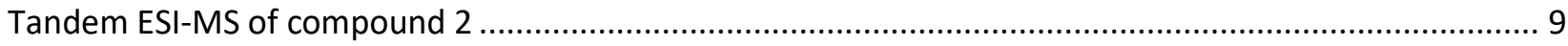

Analysis of acid hydrolysates of compound 2 and standard amino acids .............................................. 9

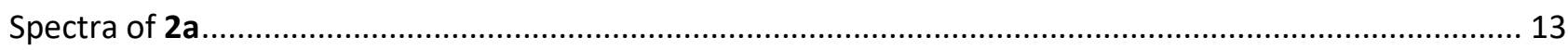

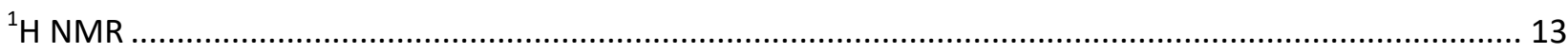

${ }^{13}$ C NMR 


HMBC 13

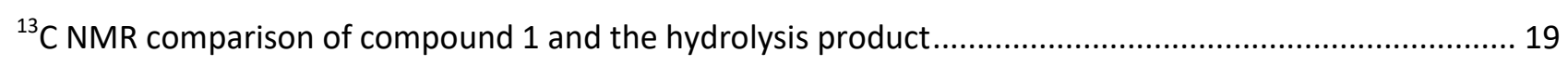




\section{Fungus Material}

The endophytic strain KY-9 was isolated from healthy leaves of Melia azedarach collected in Yangling, Shaanxi province, China. This strain was authenticated based on sequence analysis of the ITS region of rDNA (GenBank Accession No. MK396569.1, Supporting Information) listed as below. The results indicate that KY-9 was closely related to D. eucalyptorum (KACC48653), with the ITS sequence similarity of $99 \%$. Combined with morphological characteristics, the fungus was identified as D. eucalyptorum KY-9.

\section{Sequencing results:}

TGCGGAGGGATCATTGCTGGAACGCGCCCCAGGCGCACCCAGAAACCCTT

TGTGAACTTATACCTTACTGTTGCCTCGGCGCATGCCGGCCCCCTCGGGG

CCCCCTGGAGACAGGGAGCAGGCACGCCGGCGGCCAAGTTAACTCCTGTT

TTTACACTGAAACTCTGAGAAAAAACACAAATGAATCAAAACTTTCAACA

ACGGATCTCTTGGTTCTGGCATCGATGAAGAACGCAGCGAAATGCGATAA

GTAATGTGAATTGCAGAATTCAGTGAATCATCGAATCTTTGAACGCACAT

TGCGCCCTCTGGTATTCCGGAGGGCATGCCTGTTCGAGCGTCATTTCAAC

CCTCAAGCATTGCTTGGTGTTGGGGCACTGCCTGTAAAAGGGCAGGCCCT

GAAATCTAGTGGCGAGCTCGCCAGGACCCCGAGCGCAGTAGTTAAACCCT

CGCTTTGGAAGGCCCTGGCGGTGCCCTGCCGTTAAACCCCCAACTTCTGA

AAATTTGACCTCGGATCAGGTAGGAATACCCGCTGAACTTAAGCATATC

[Refer.] S. X. Zhang, H. Fang, C. P. Yin, J. W. Hu, C. L. Wei, Y. L. Zhang. Antimicrobial metabolites produced by Penicillium mallochii CCH01 isolated from the gut of Ectropis oblique, cultivated in the presence of a histone deacetylase inhibitor. Front. Microbiol. 2019, 10:2186.

\section{Spectra of compound 1}

\section{${ }^{1}$ H NMR}

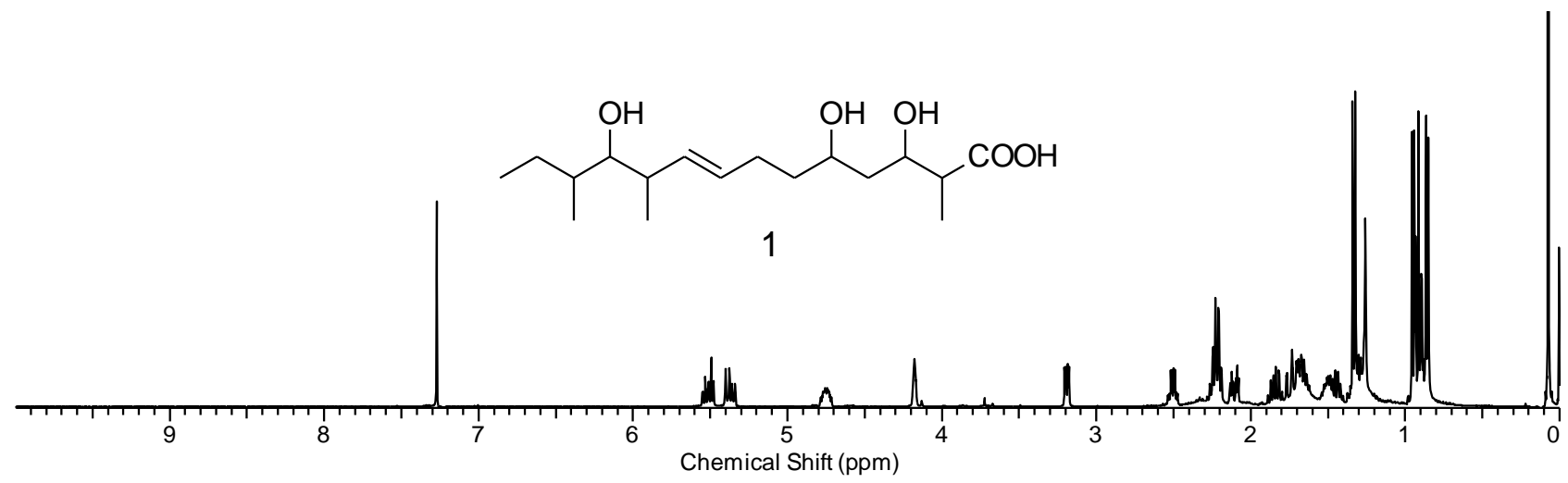


${ }^{13}$ C NMR

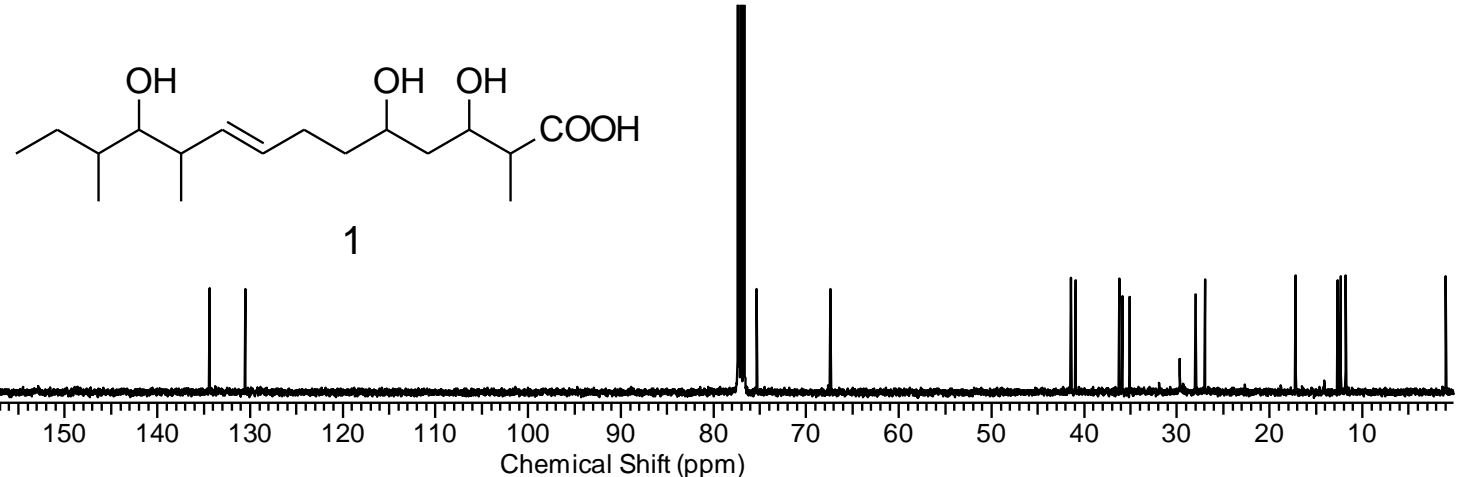

\section{HSQC}

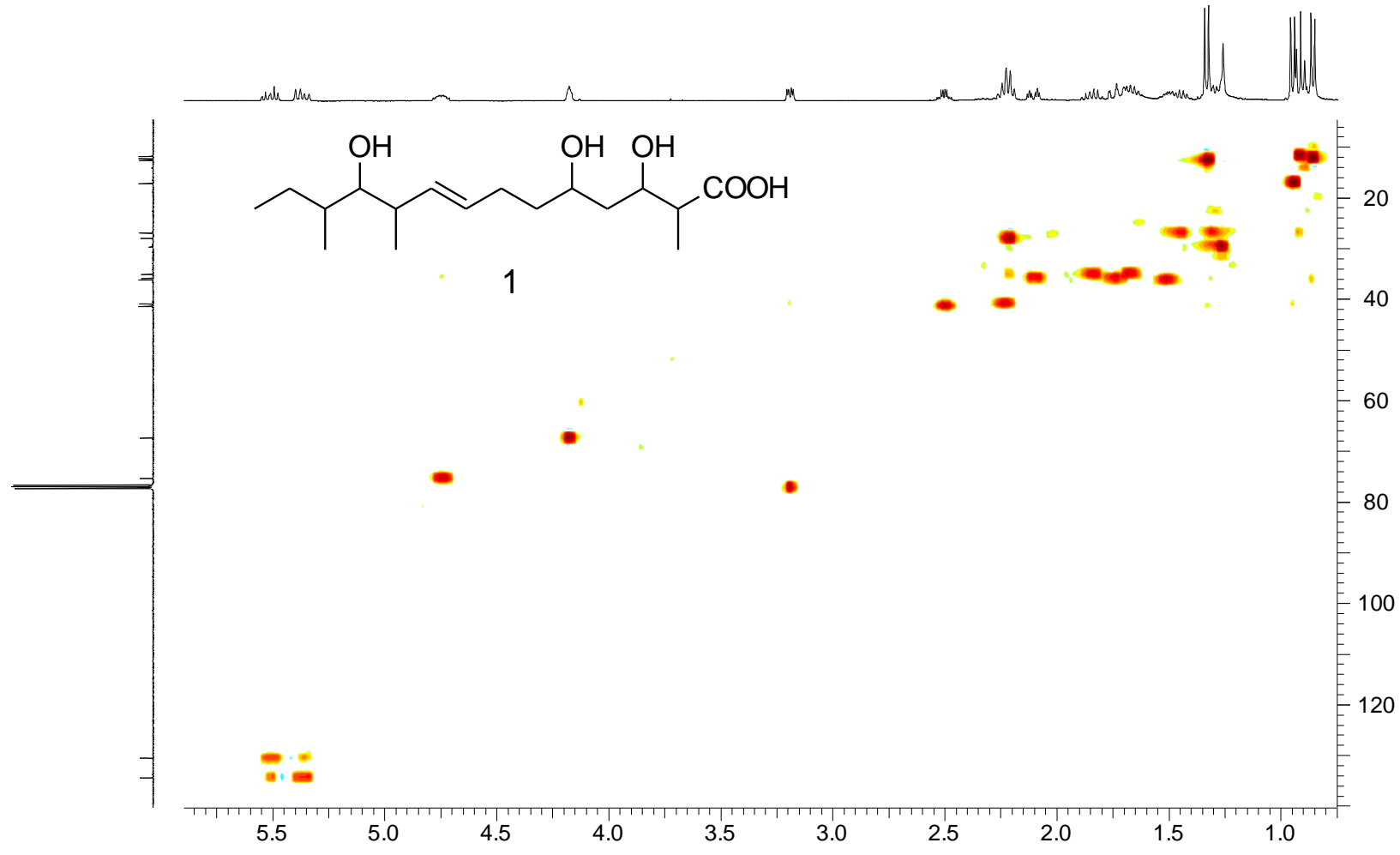




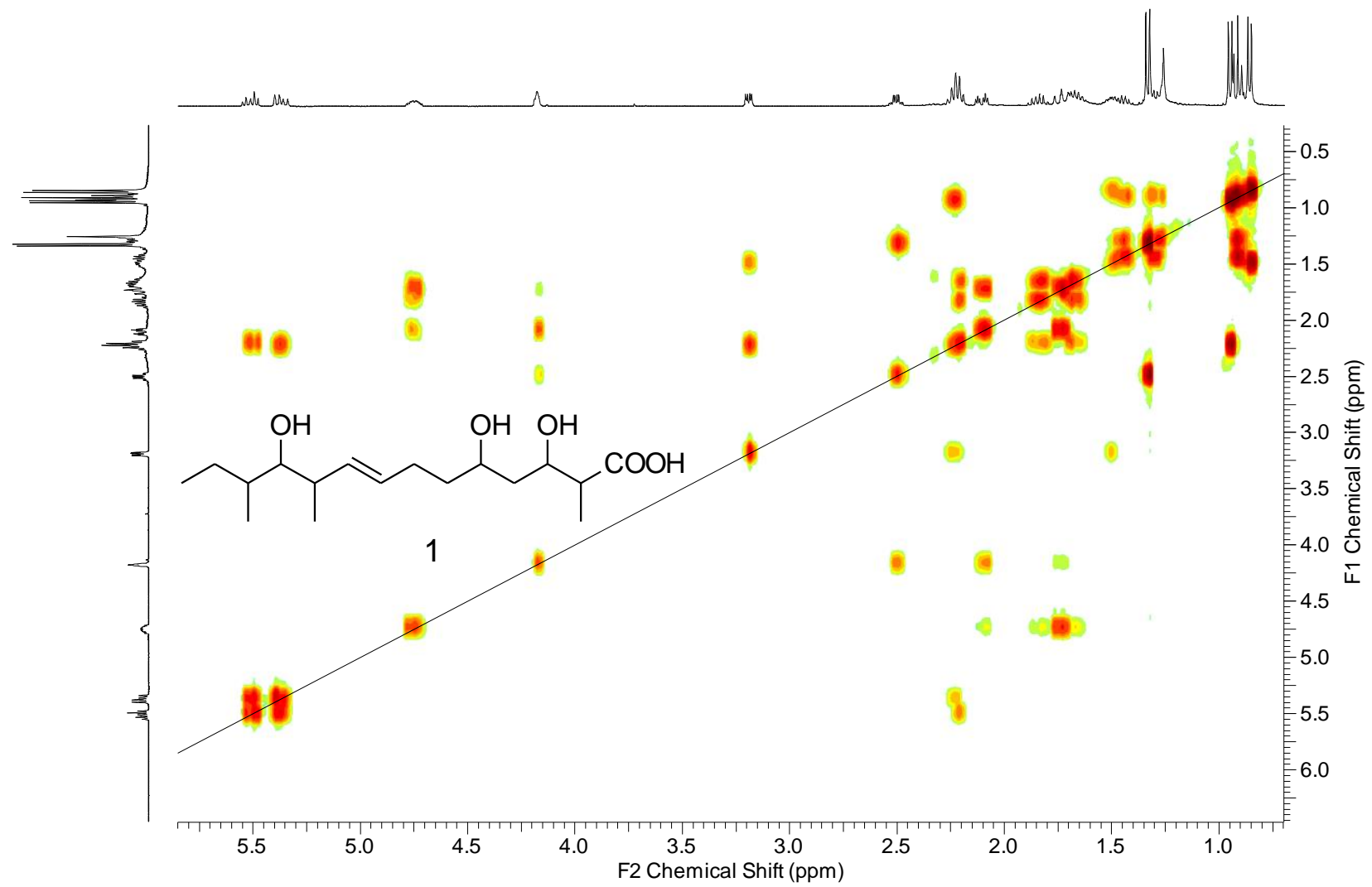

\section{HMBC}

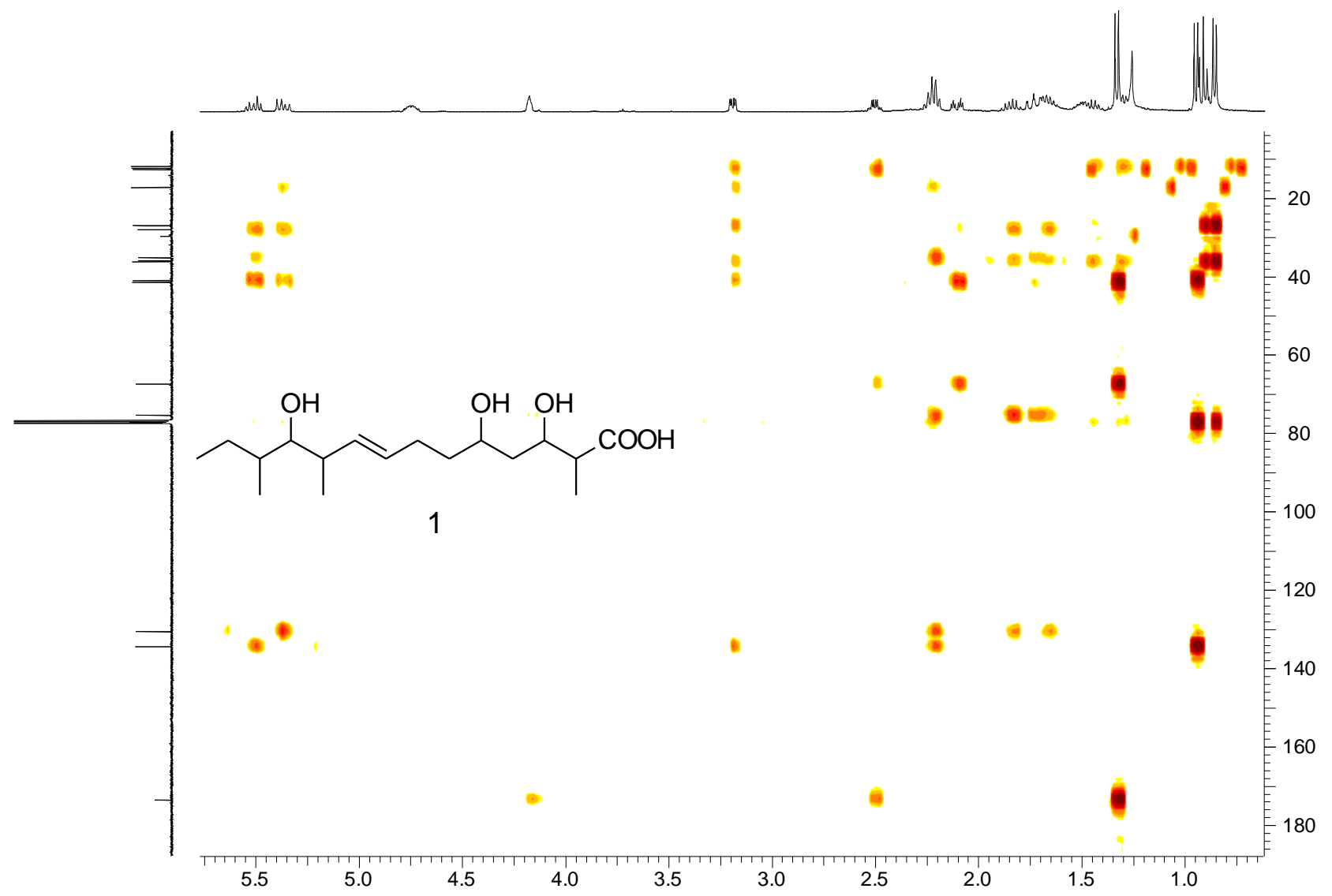


HRMS

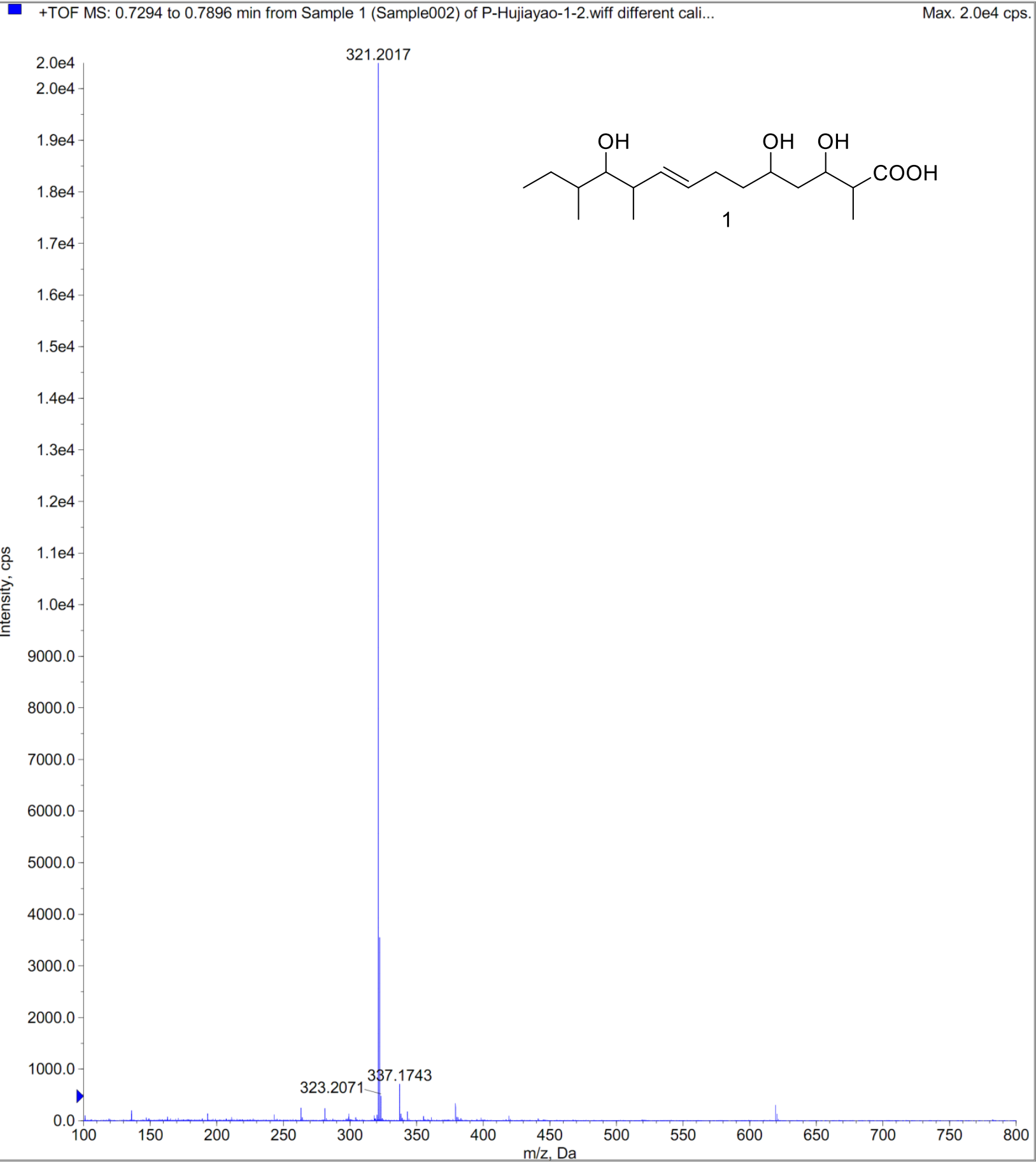


Spectra of compound 2

\section{${ }^{1}$ H NMR}

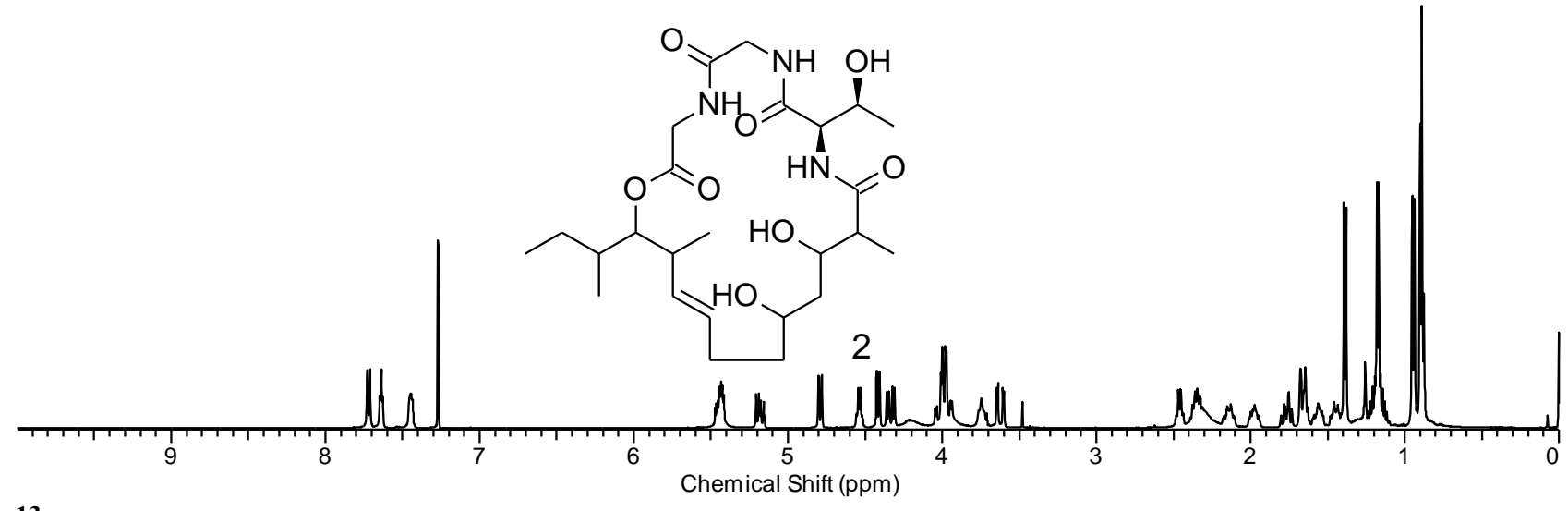

${ }^{13}$ C NMR

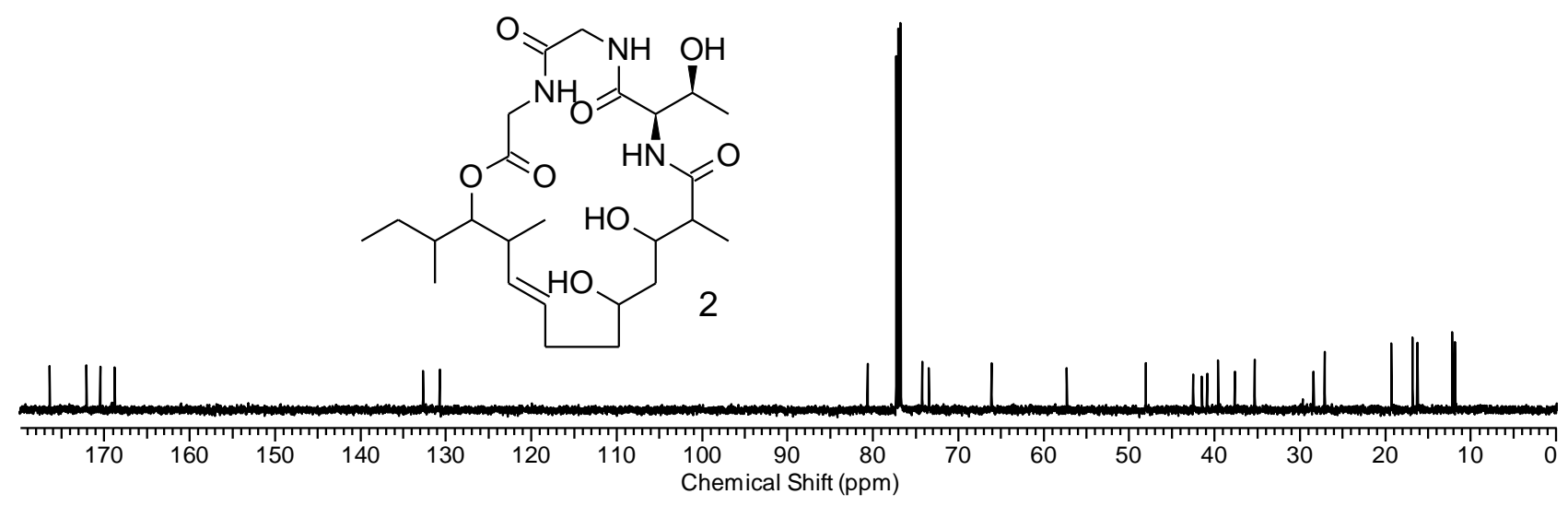

\section{HSQC}

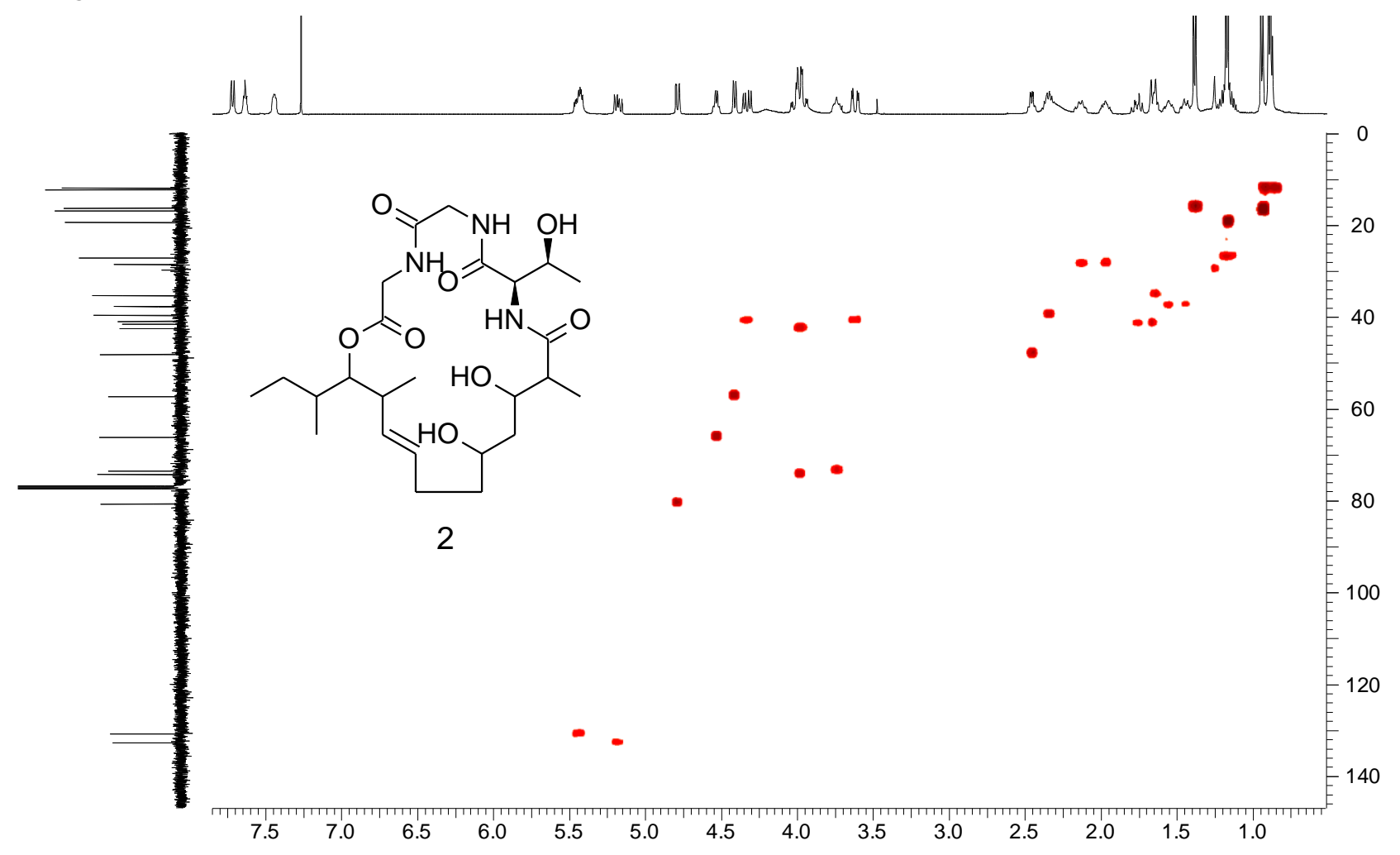




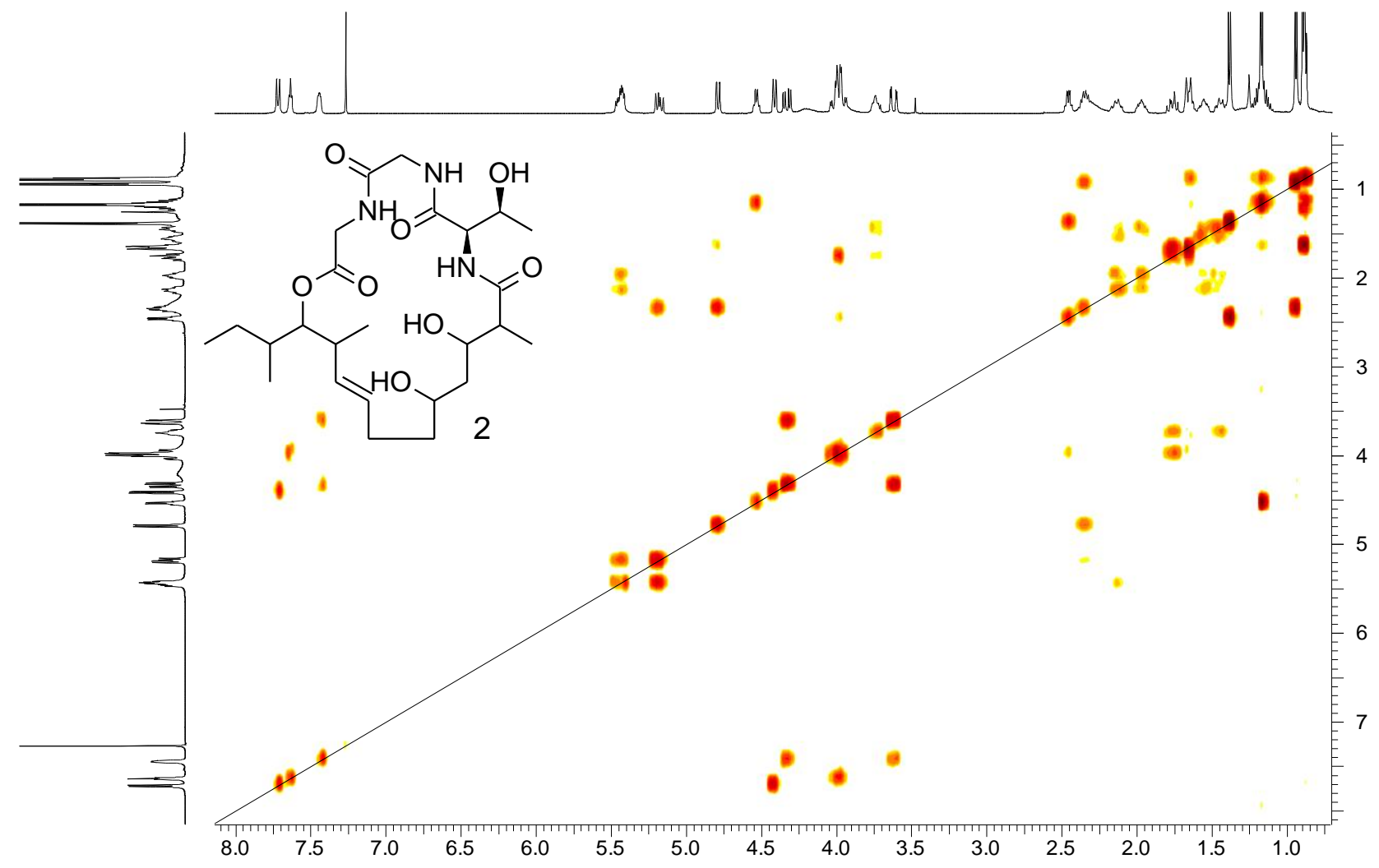

\section{HMBC}

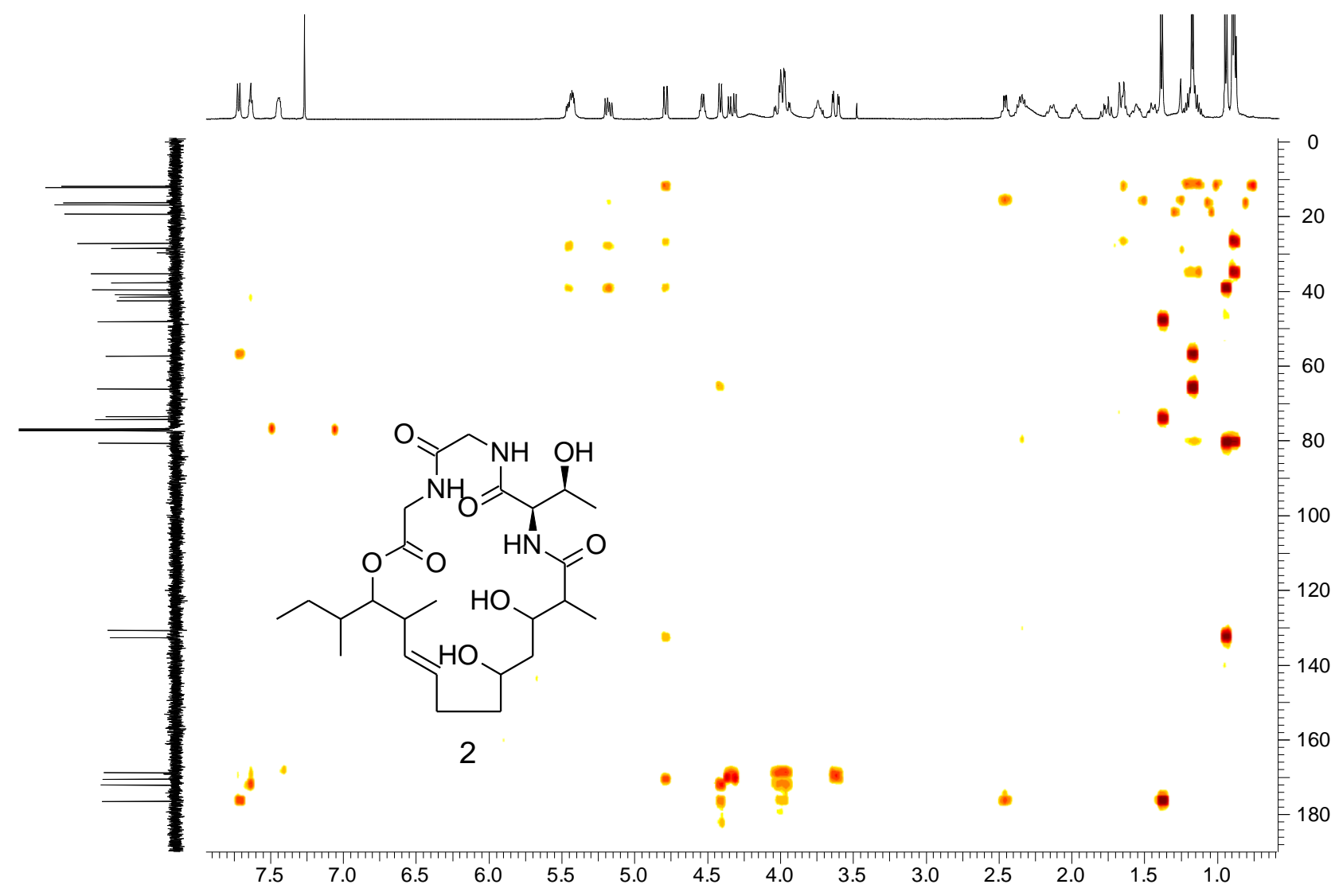




\section{NOESY}

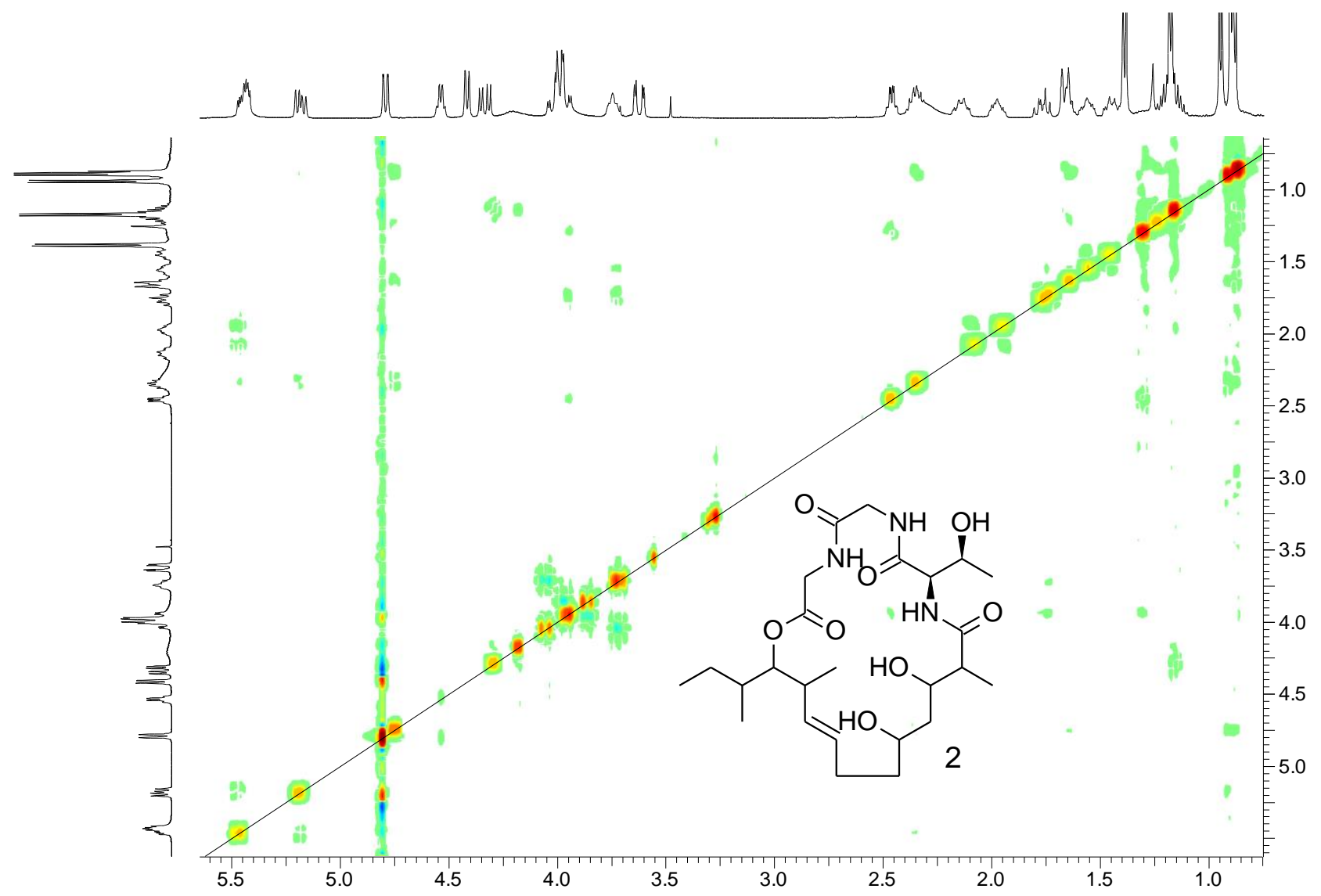

\section{HRMS of 2}

Event\#: 3 MS(E-) Ret. Time : 0.257 -> 0.587 Scan\# : 59 -> 131

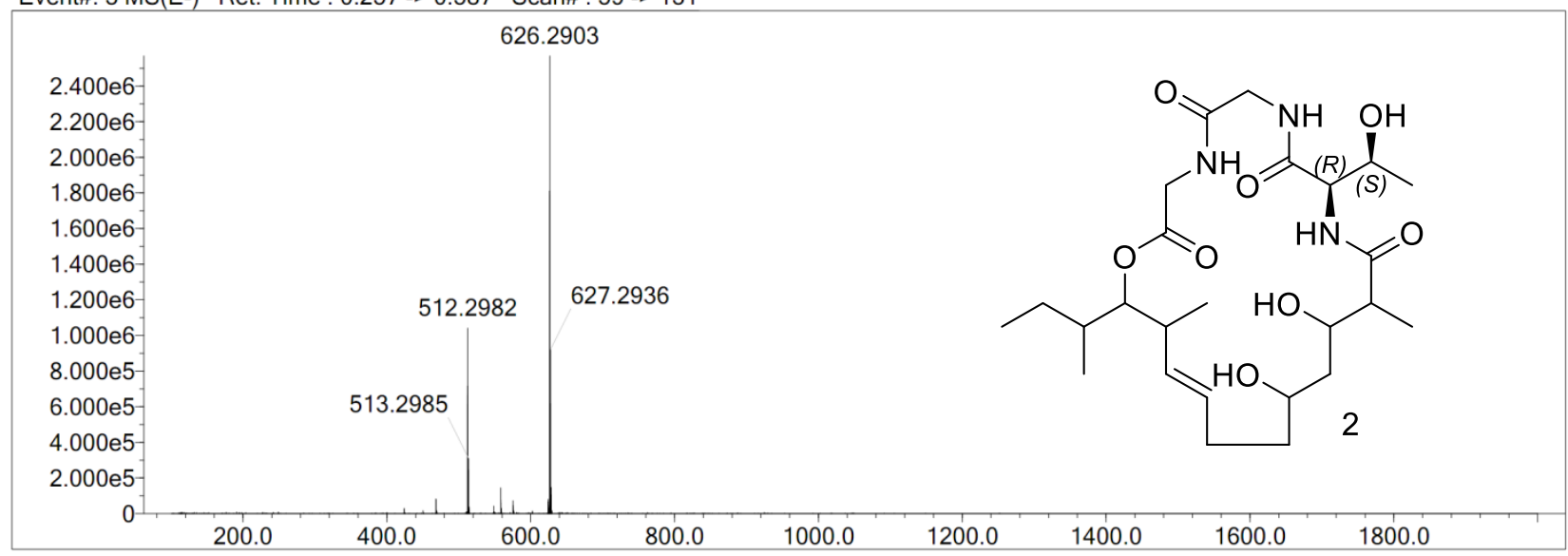

\section{Tandem ESI-MS of compound 2}

\section{Negative mode}

Full MS: $m / z 512[\mathrm{M}-\mathrm{H}]^{-}, m / z 548[\mathrm{M}+\mathrm{Cl}]^{-}$ 


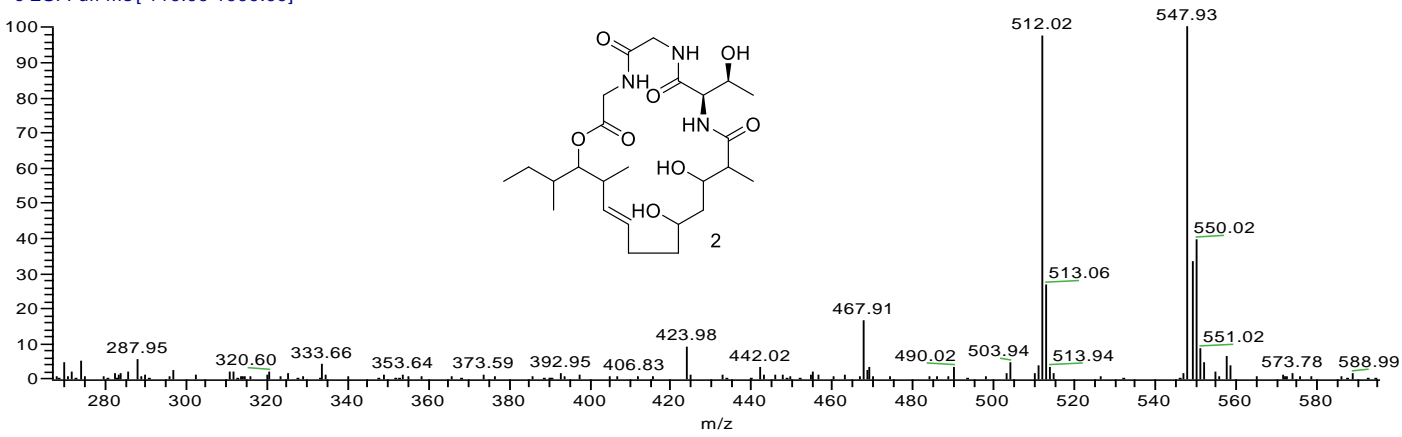

(2) $\mathrm{MS}^{2}$ of $m / z 512$

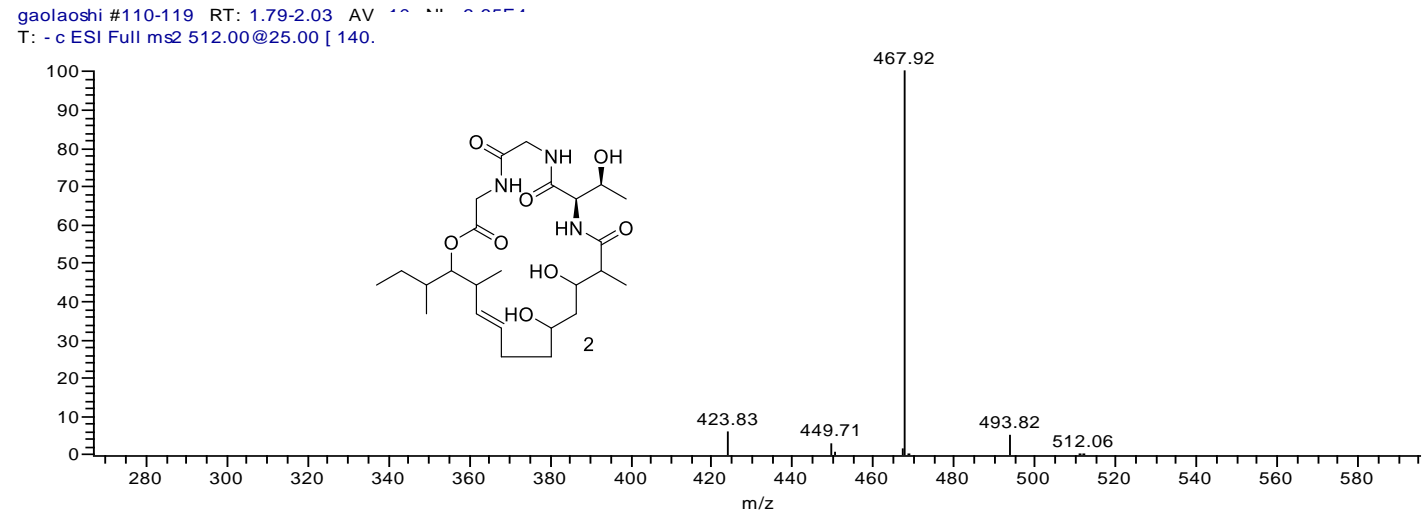

(3) $\mathrm{MS}^{2}$ of $m / z 548$

gaolaoshi \#190-195 RT: 4.15-4.28 AV . nu - n ntrn

T: - c ESI Full ms2 $548.00 @ 25.00[150$

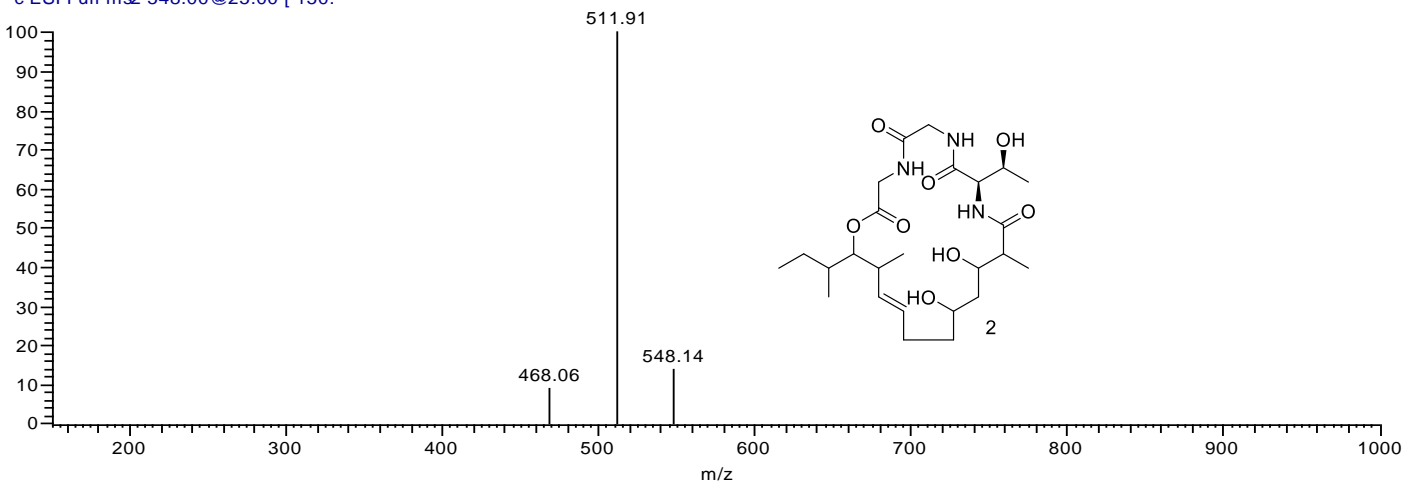

(4) $\mathrm{MS}^{3}$ of $m / z 512$ based on $m / z, 468$

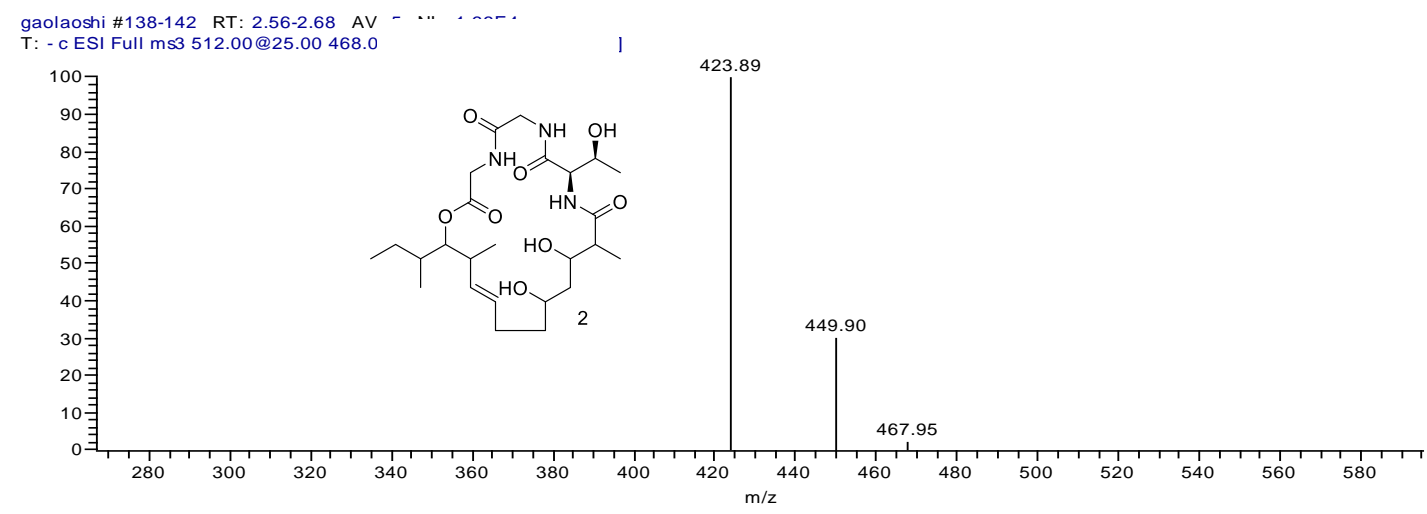




\section{Positive mode}

\section{(1) Full MS, $m / z 536[\mathrm{M}+\mathrm{Na}]^{+}$}

gaolaoshi \#250-260 RT: 5.59-5.75 AV . + ... . . n.

T: + c ESI Full ms [ 140.00-1000.00]

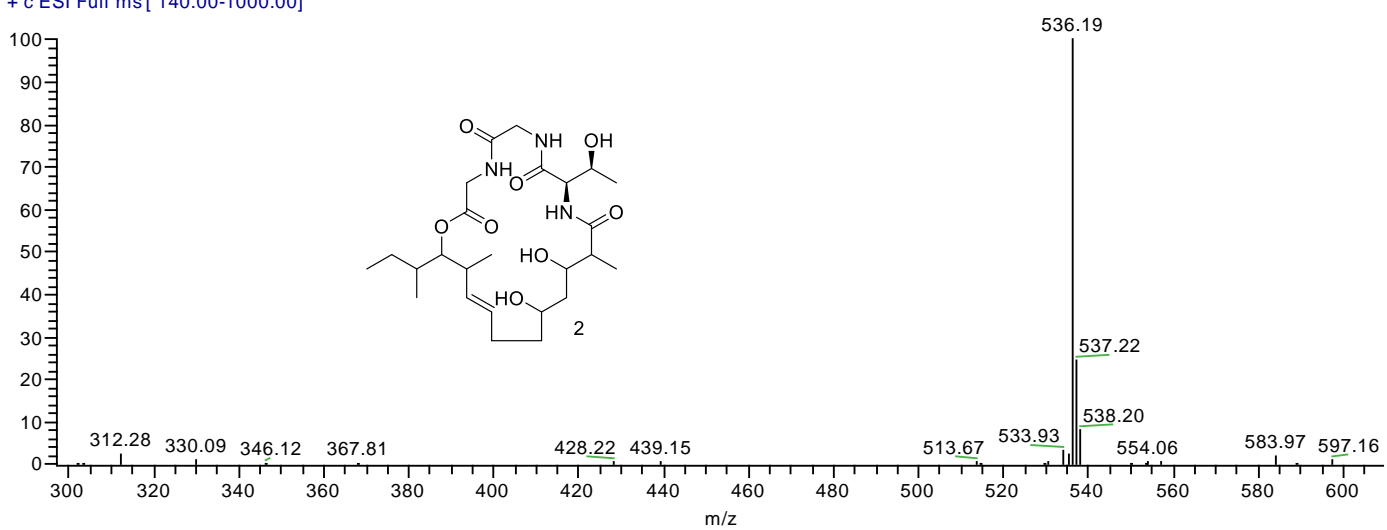

\section{(2) $\mathrm{MS}^{2}$ of $m / z 536$}

gaolaoshi \#337-342 RT: 7.59-7.72 AV
T: + c ESI Full ms2 536.00@40.00 [ 145
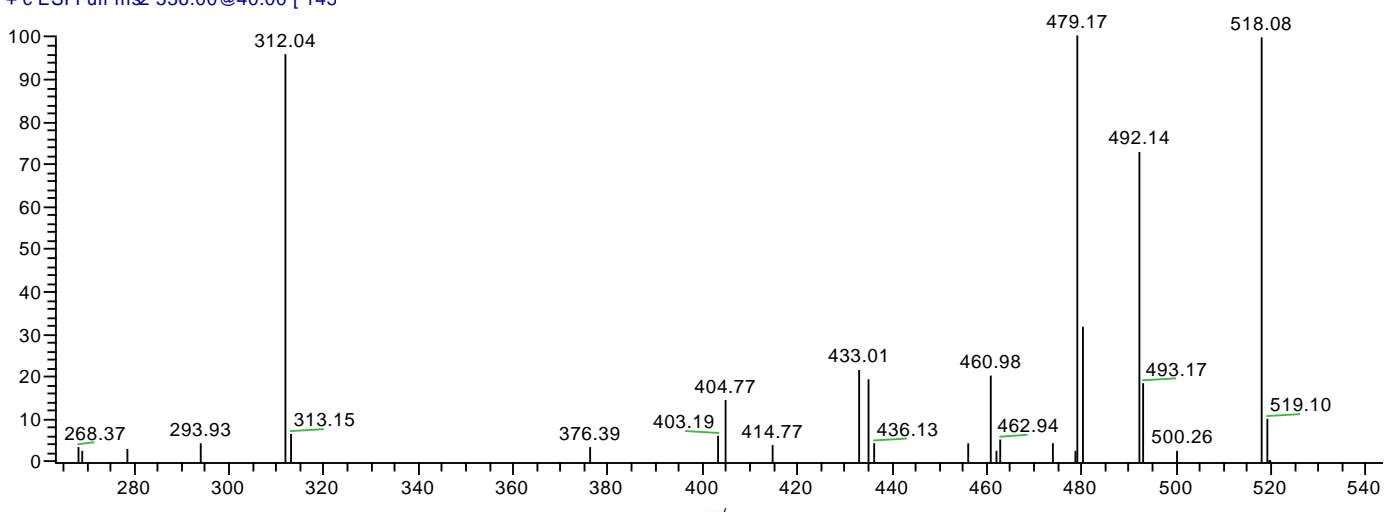
3. Proposed fragmentation pathways of compound 2

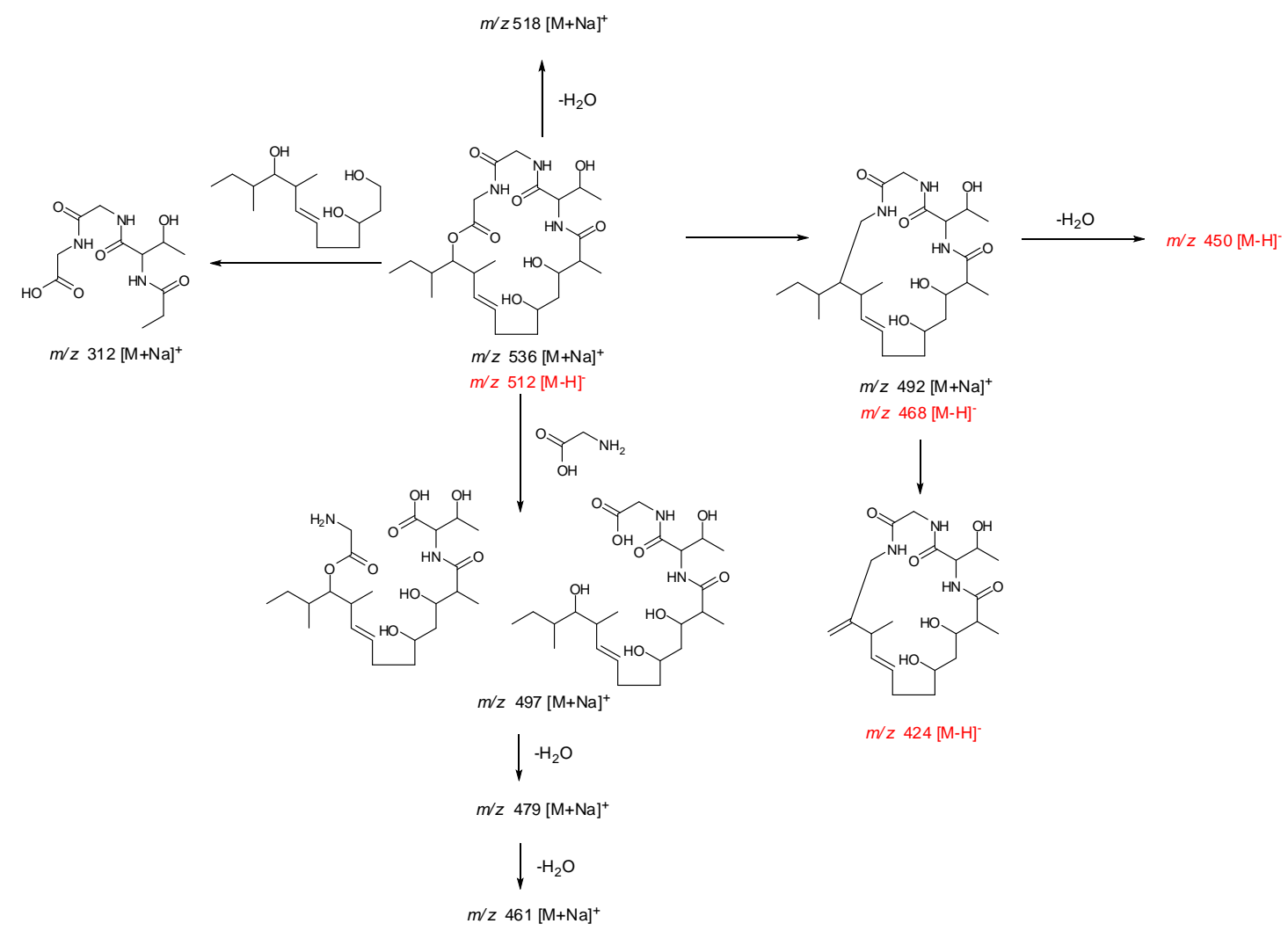

4. Analysis of acid hydrolysates of compound 2 and standard amino acids

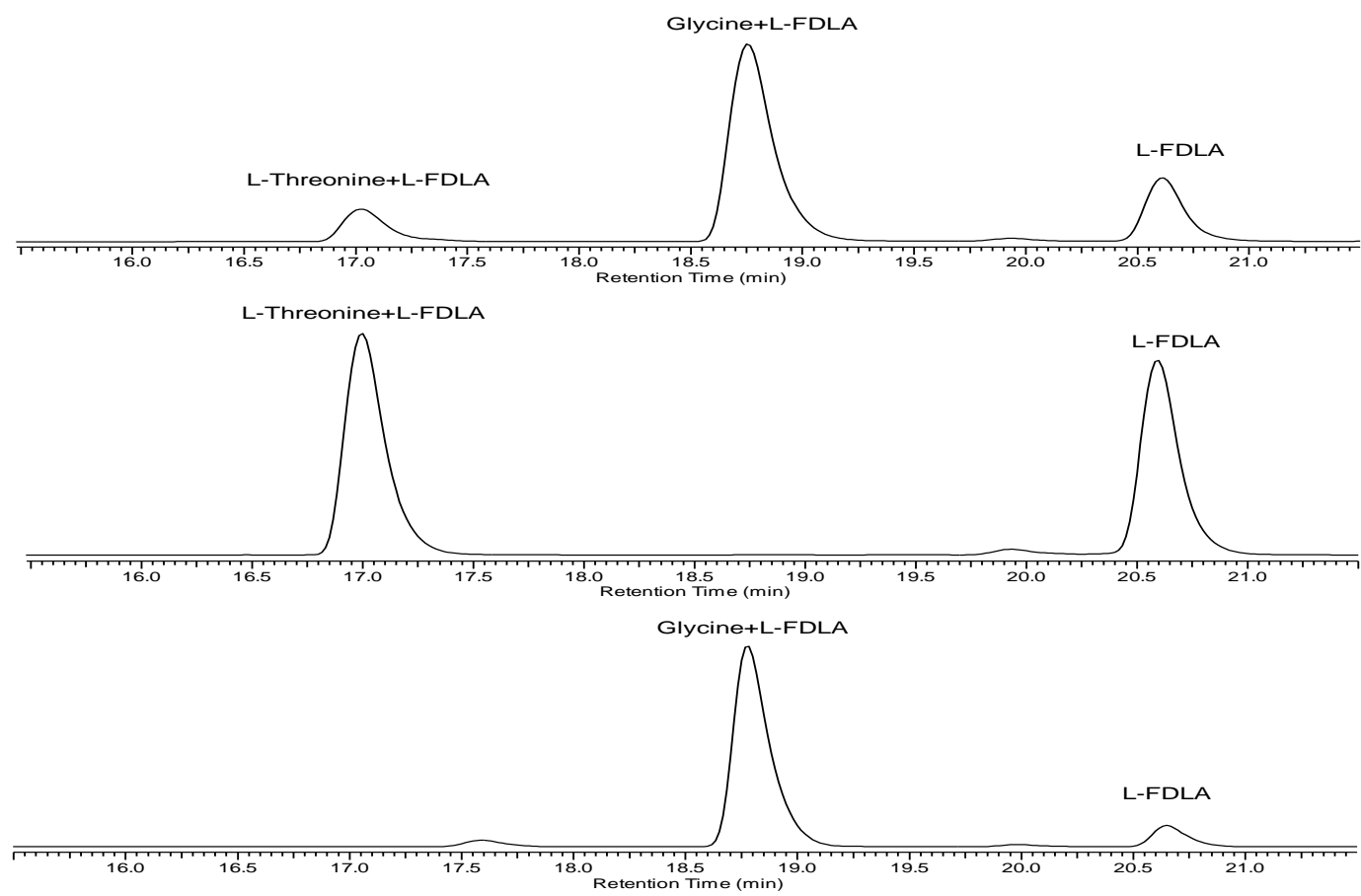


Spectra of $2 a$

\section{${ }^{1}$ H NMR}

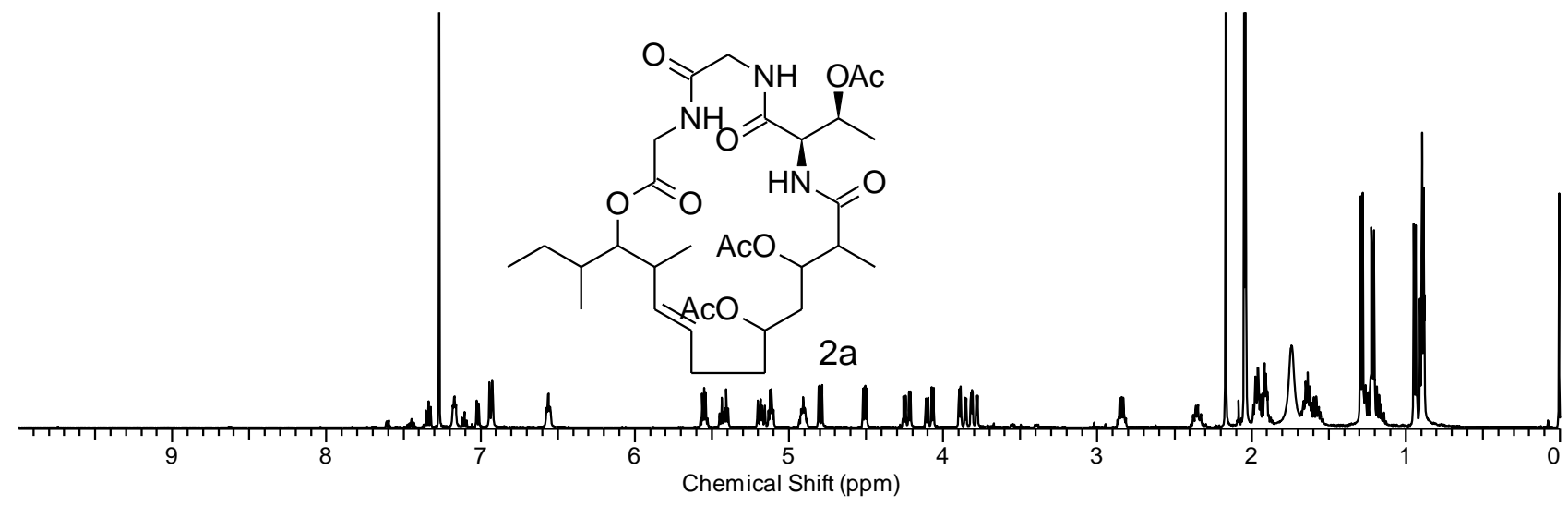

${ }^{13}$ C NMR

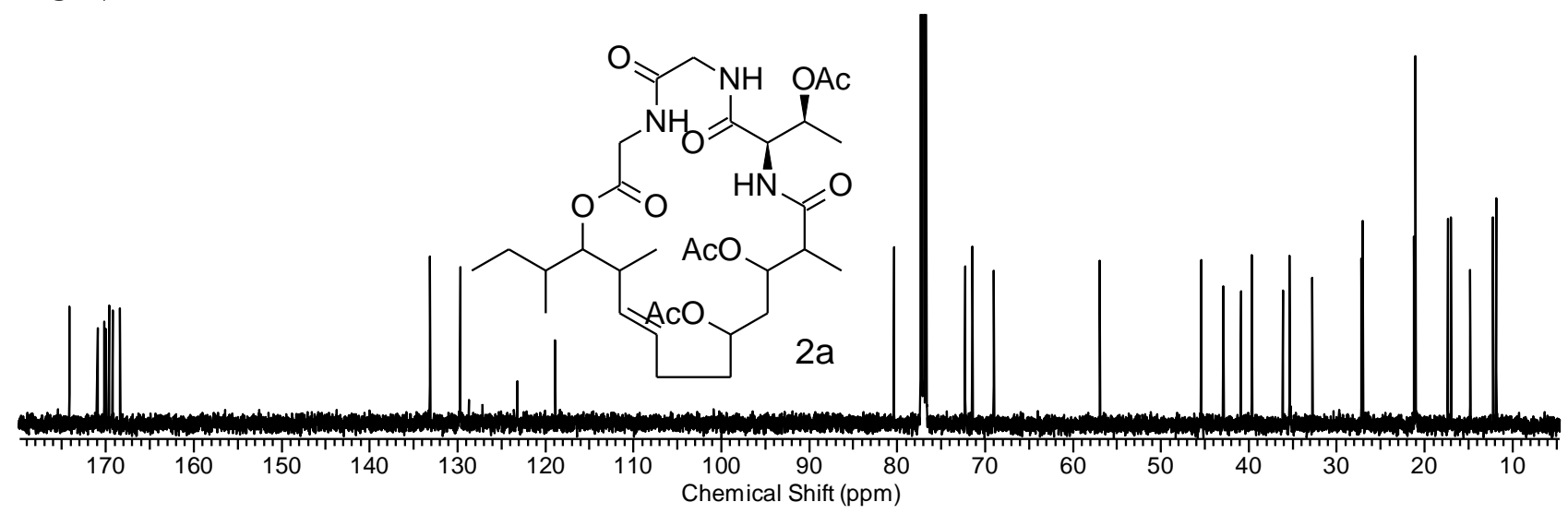

\section{HSQC}

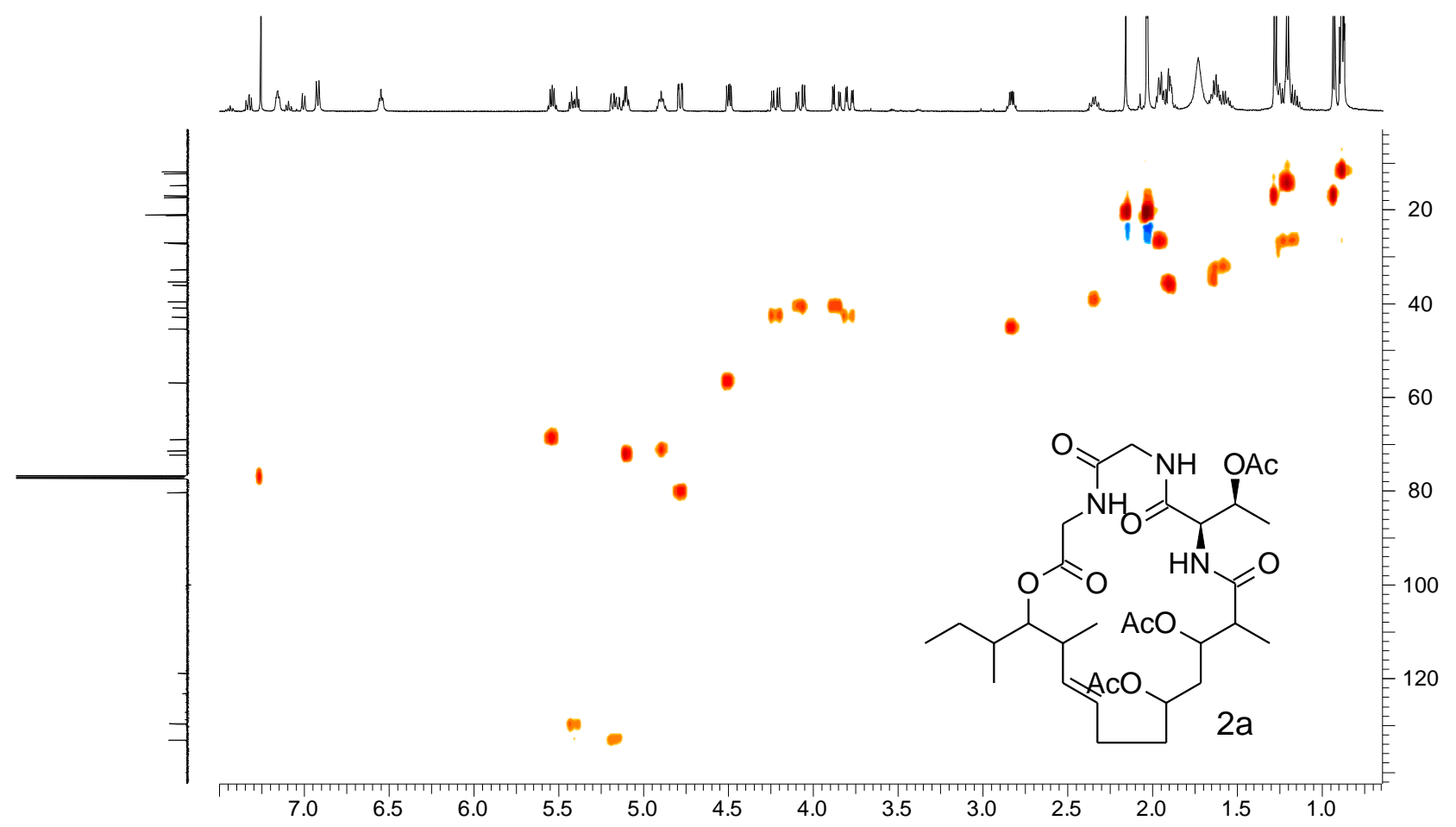


HMBC

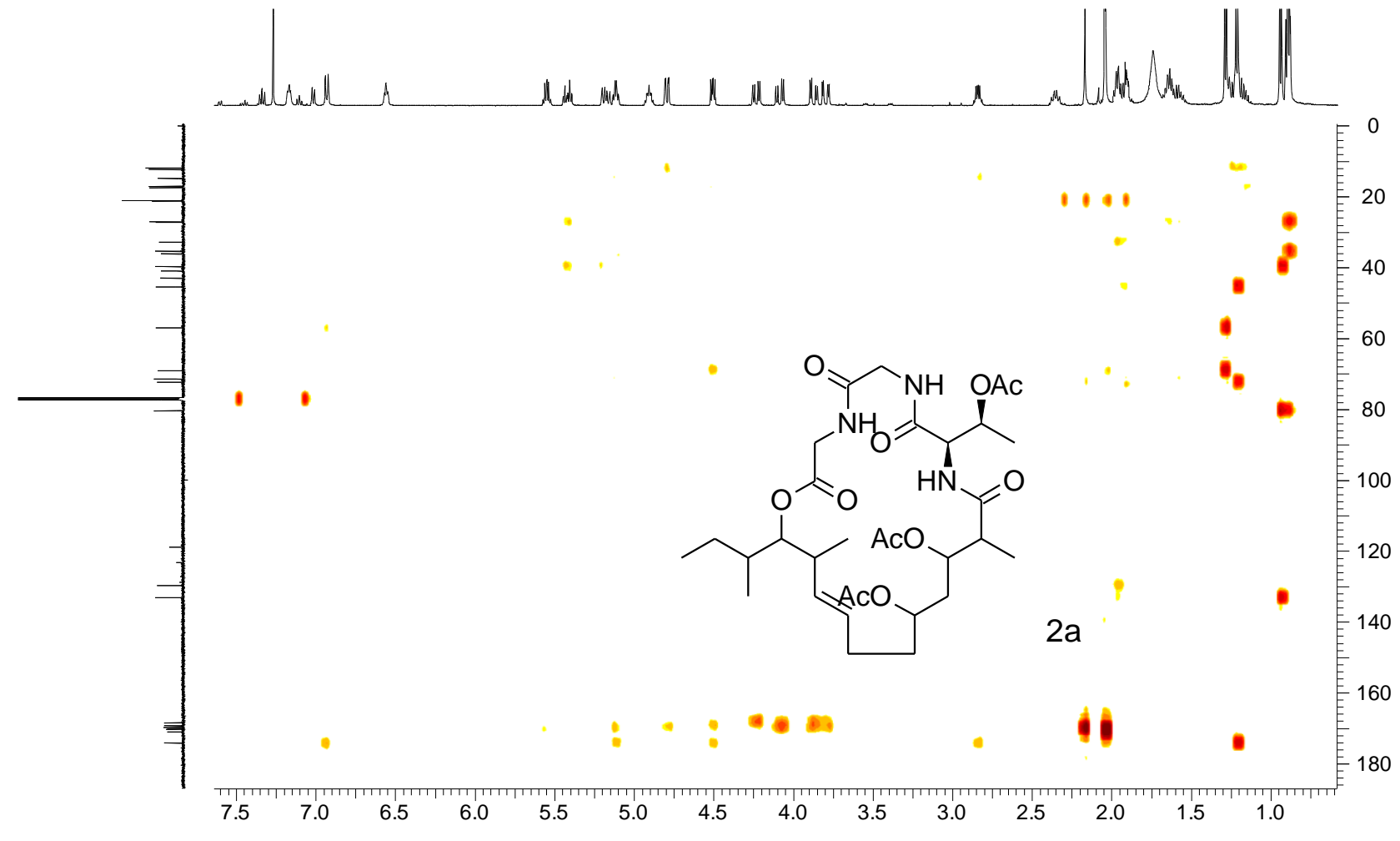


HRMS of $2 a$

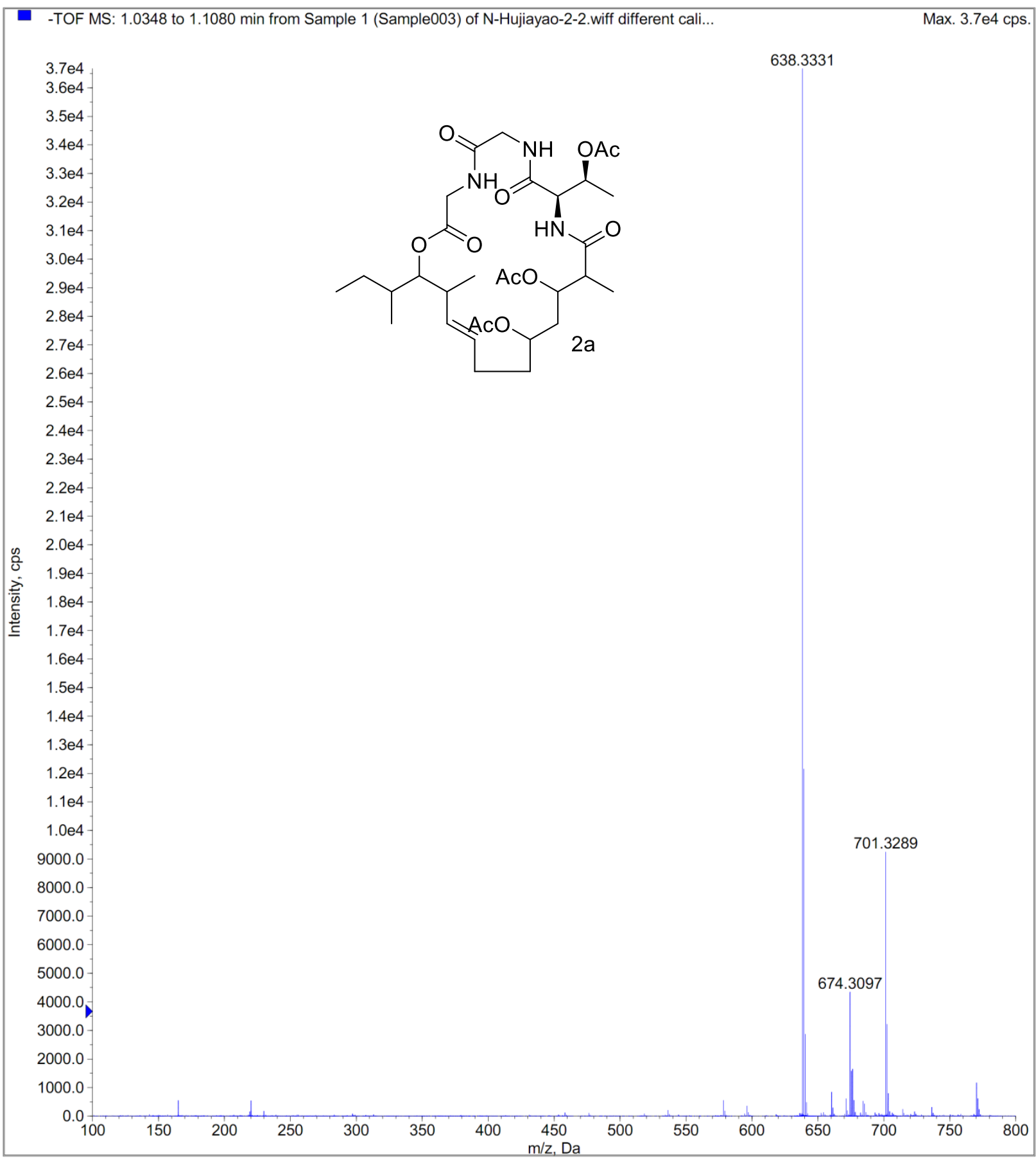




\section{Spectra of 2b}

\section{${ }^{1}$ H NMR}

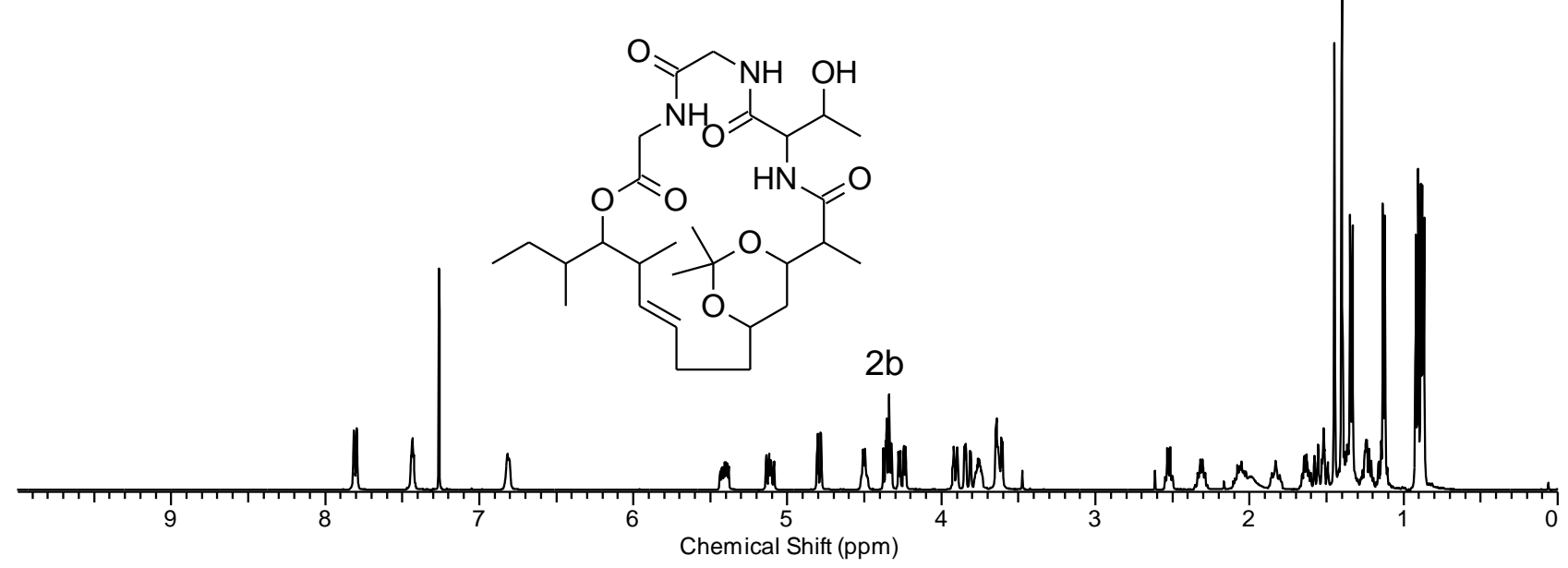

${ }^{13}$ C NMR

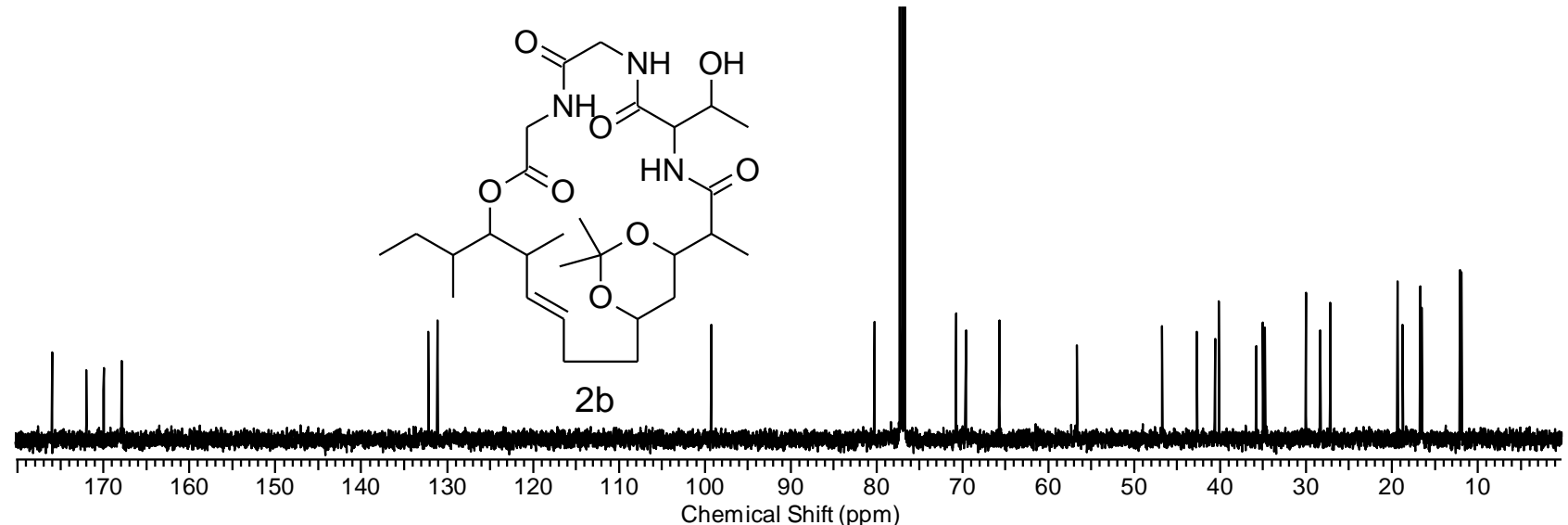

\section{HSQC}

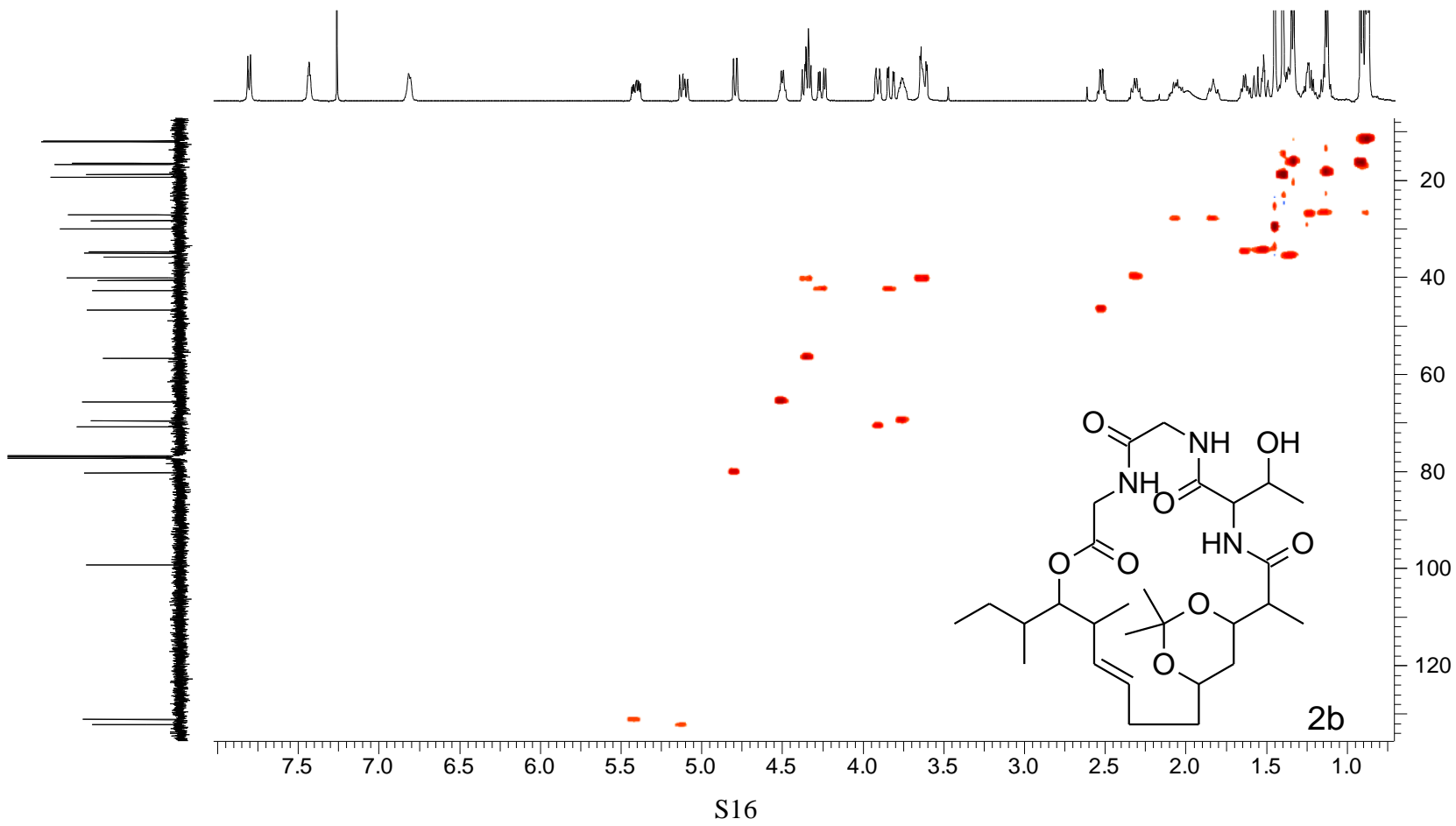




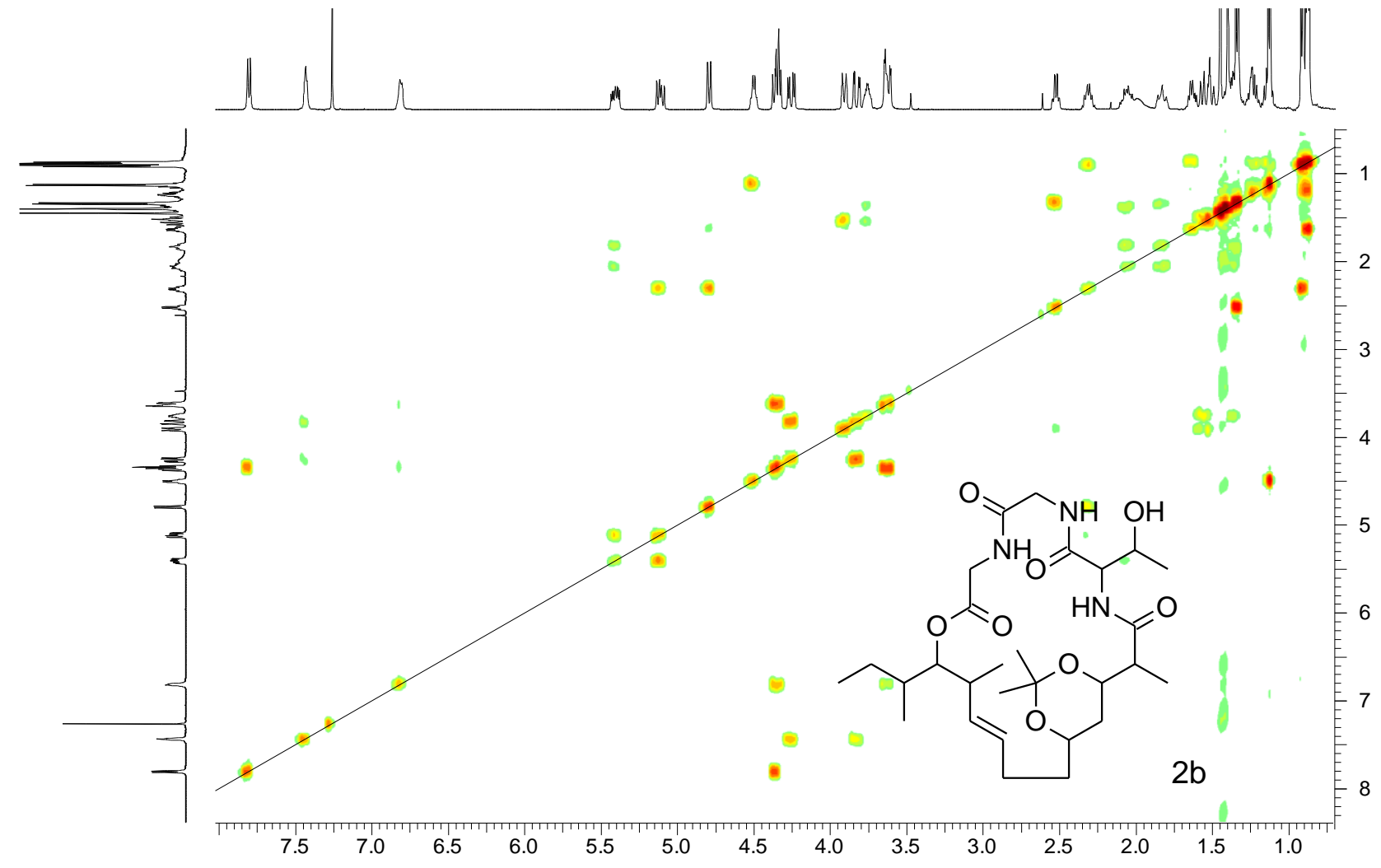

\section{HMBC}

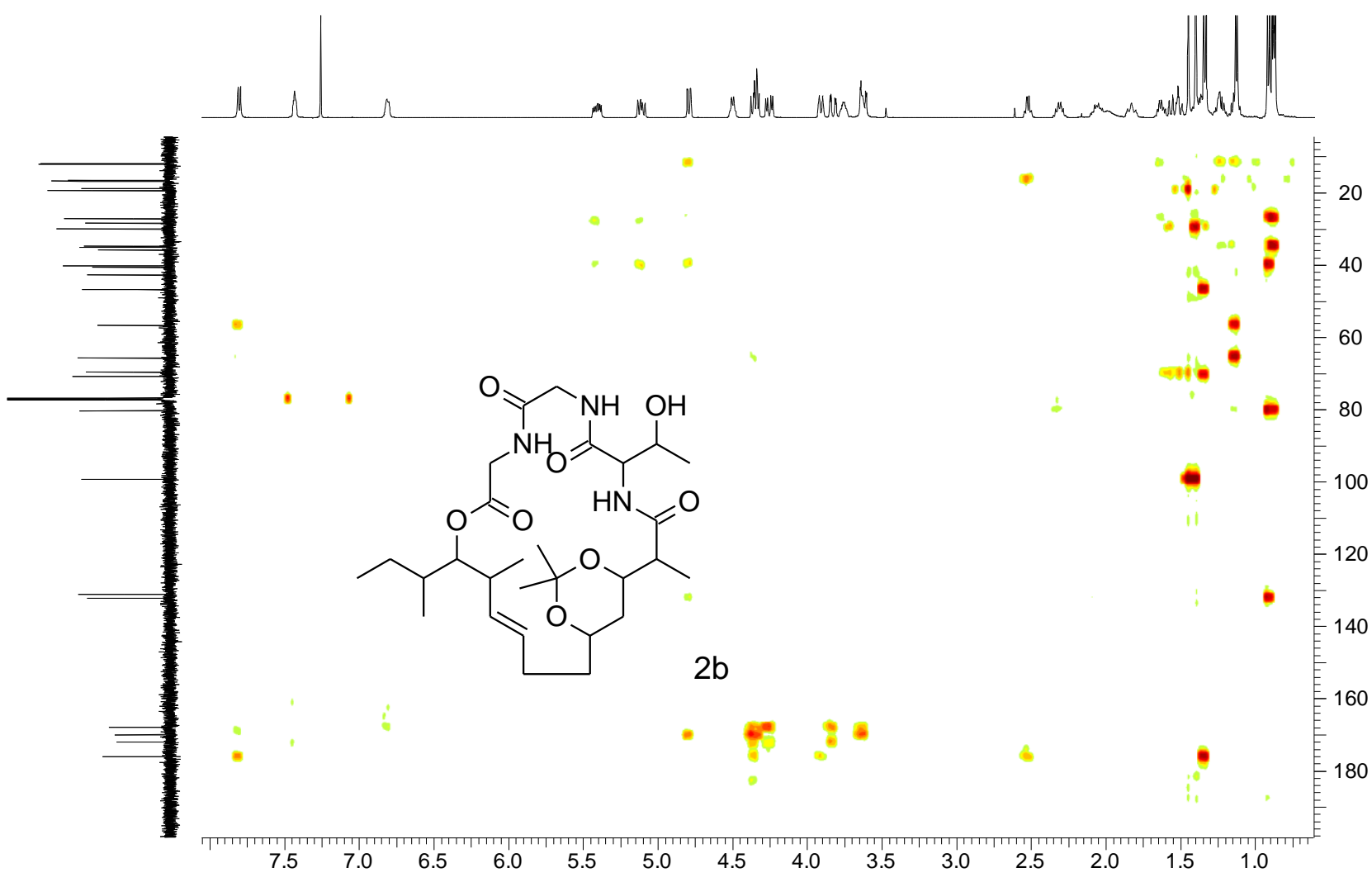




\section{NOESY}

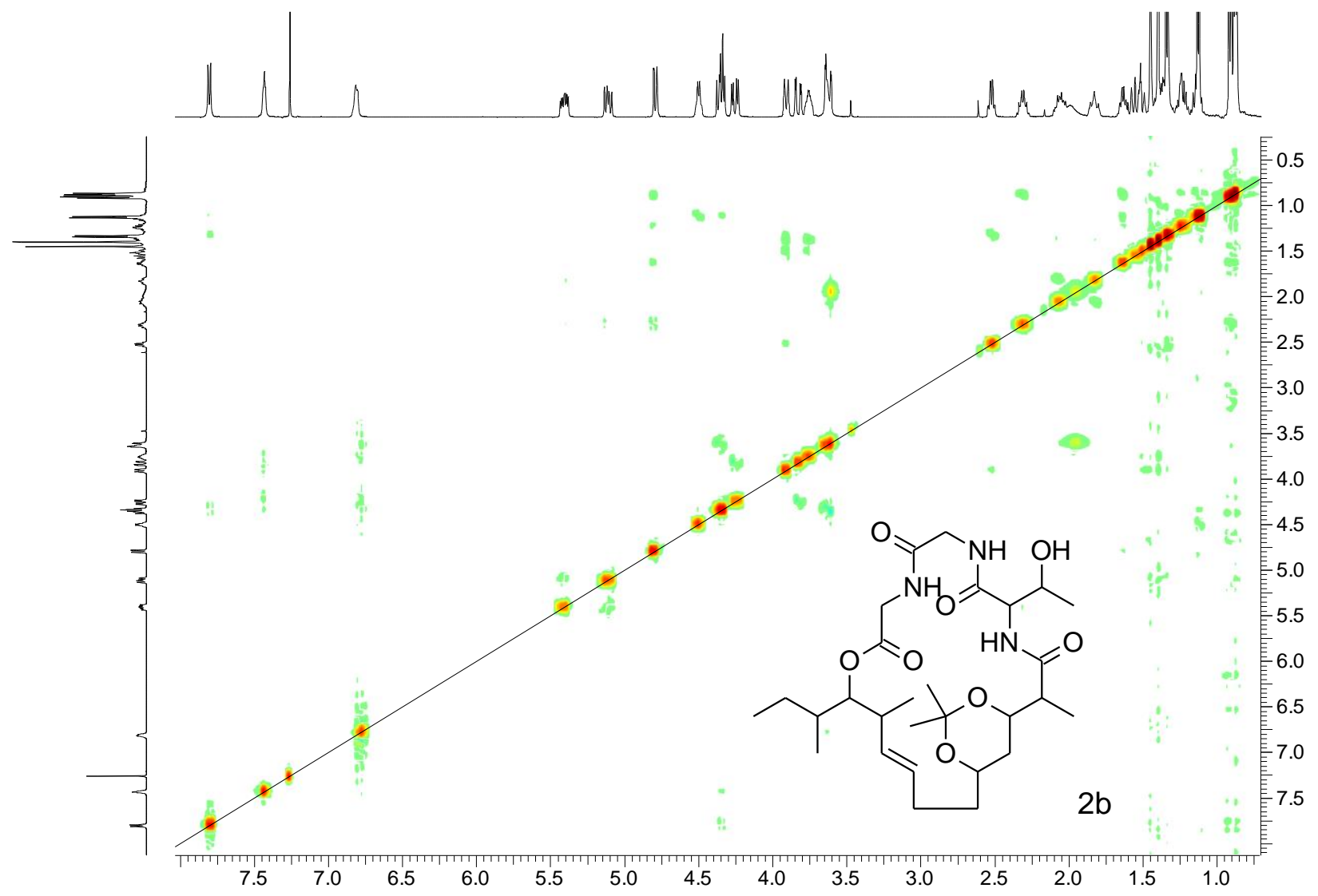

HRMS of $2 b$

Event\#: 3 MS(E-) Ret. Time : 0.275 -> 0.587 - 0.917 -> 1.742 Scan\# : 63 -> 131 - 203 -> 383

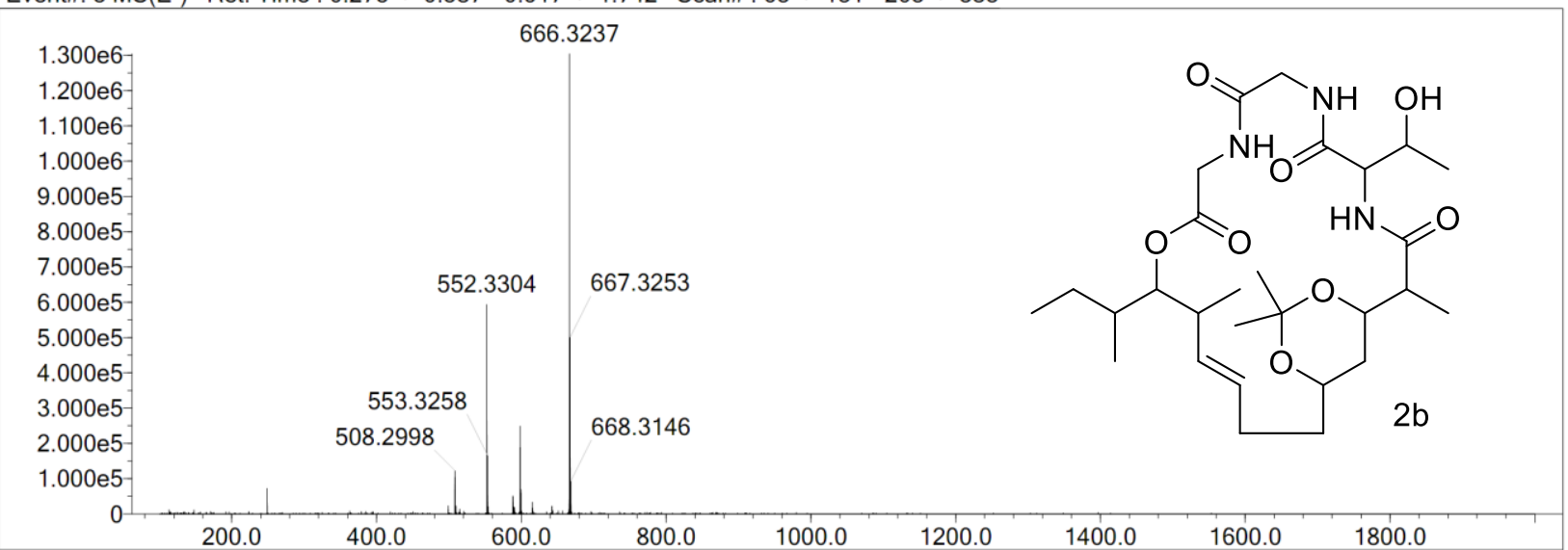


${ }^{13} \mathrm{C}$ NMR comparison of compound 1 and the hydrolysis product

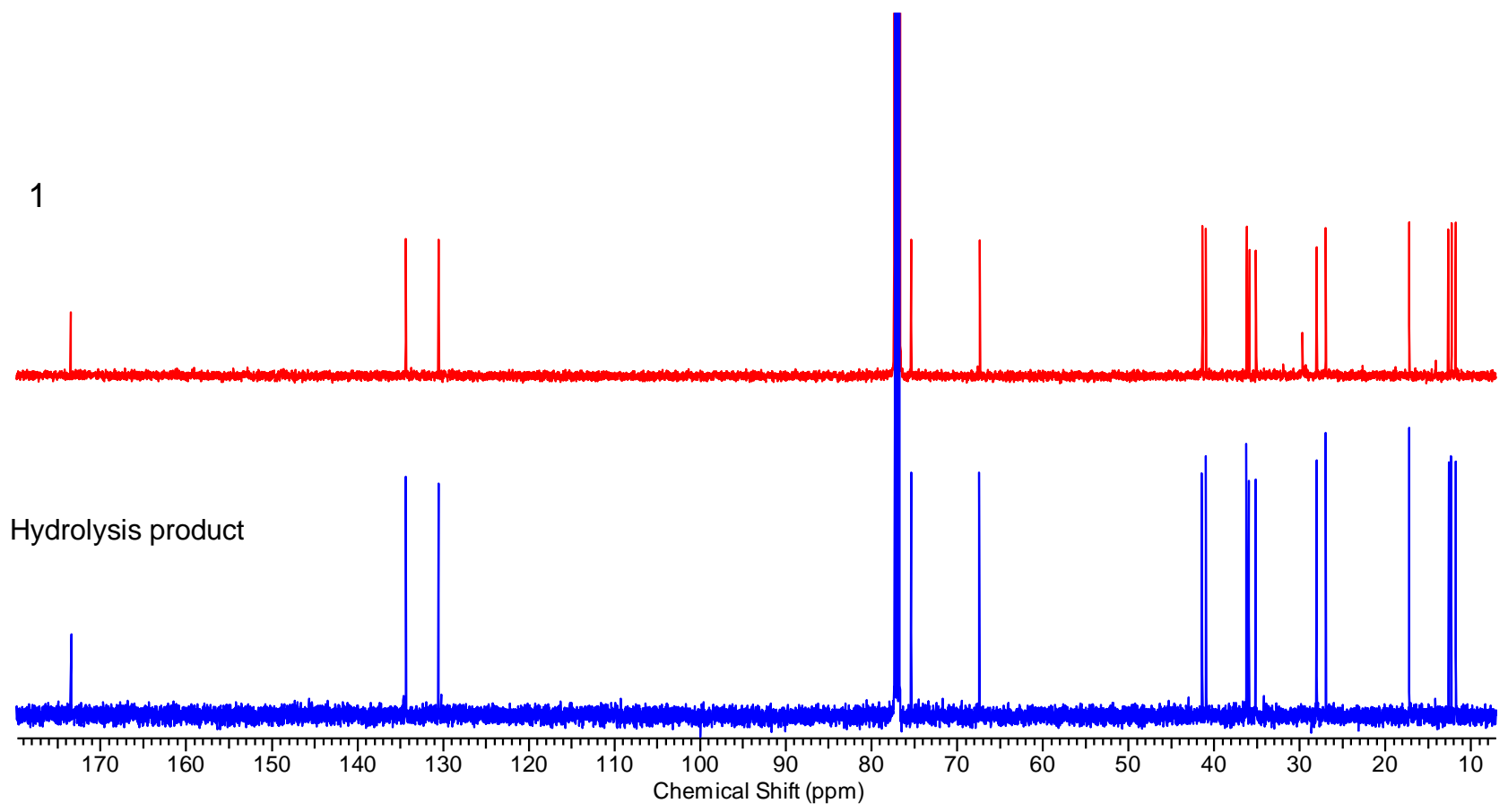

